\title{
Comparison of Current Methods for Signal Peptide Prediction in Phytoplasmas
}

\author{
Christophe Garcion*, Laure Béven and Xavier Foissac \\ INRAE, Univ. Bordeaux, Biologie du Fruit et Pathologie, UMR 1332, Villenave d'Ornon, France
}

Although phytoplasma studies are still hampered by the lack of axenic cultivation methods, the availability of genome sequences allowed dramatic advances in the characterization of the virulence mechanisms deployed by phytoplasmas, and highlighted the detection of signal peptides as a crucial step to identify effectors secreted by phytoplasmas. However, various signal peptide prediction methods have been used to mine phytoplasma genomes, and no general evaluation of these methods is available so far for phytoplasma sequences. In this work, we compared the prediction performance of SignalP versions 3.0, 4.0, 4.1, 5.0 and Phobius on

OPEN ACCESS

Edited by:

Chih-Horng Kuo,

Institute of Plant and Microbial

Biology, Academia Sinica, Taiwan

Reviewed by:

Cristina Marzachi,

Istituto per la Protezione Sostenibile delle Piante (CNR), Italy

Kenro Oshima,

Hosei University, Japan

*Correspondence:

Christophe Garcion

christophe.garcion@inrae.fr

Specialty section:

This article was submitted to

Evolutionary and Genomic

Microbiology,

a section of the journal

Frontiers in Microbiology

Received: 30 January 2021 Accepted: 02 March 2021

Published: 25 March 2021

Citation:

Garcion C, Béven $L$ and Foissac X

(2021) Comparison of Current Methods for Signal Peptide Prediction

in Phytoplasmas.

Front. Microbiol. 12:661524.

do: $10.3389 /$ fmicb.2021.661524 several sequence datasets originating from all deposited phytoplasma sequences. SignalP 4.1 with specific parameters showed the most exhaustive and consistent prediction ability. However, the configuration of SignalP 4.1 for increased sensitivity induced a much higher rate of false positives on transmembrane domains located at $\mathrm{N}$-terminus. Moreover, sensitive signal peptide predictions could similarly be achieved by the transmembrane domain prediction ability of TMHMM and Phobius, due to the relatedness between signal peptides and transmembrane regions. Beyond the results presented herein, the datasets assembled in this study form a valuable benchmark to compare and evaluate signal peptide predictors in a field where experimental evidence of secretion is scarce. Additionally, this study illustrates the utility of comparative genomics to strengthen confidence in bioinformatic predictions.

Keywords: bacteria, Mollicutes, SignalP, TMHMM, Phobius, secretion, effector, transmembrane domain

\section{INTRODUCTION}

Phytoplasmas are bacterial phloem-limited pathogens transmitted by insect vectors that generate diseases in infected plants. A large number of crops as well as ornamental plants and trees can be affected, showing altered development and yield losses (Lee et al., 2000). Phytoplasmas belong to the class Mollicutes, that derives from a Gram-positive, Clostridium- or Lactobacillusrelated ancestor, but whose members are distinguished by the absence of a cell wall and are delimited by their plasma membrane (Lee et al., 2000; Hogenhout et al., 2008). Within Mollicutes, phytoplasmas are rather distant from mycoplasmas, and their closest relatives are bacteria from the Acholeplasma branch. Currently axenic cultivation methods of phytoplasmas are not yet available despite numerous attempts. As a consequence, many aspects of the biology of phytoplasmas are still to be explored. In silico analyses of phytoplasma genomes allowed to identify putative virulence factors, and three phytoplasma effectors (defined as proteins produced by pathogens that alter the 
physiology of the host) and their homologs have been the focus of several recent studies. Tengu is a short protein that induces dwarfism and witches' broom, together with a downregulation of the jasmonic acid and auxin pathways (Hoshi et al., 2009; Minato et al., 2014). Remarkably, it was also shown to suppress induced cell death (Wang et al., 2018b) and to be processed by host proteases (Sugawara et al., 2013). The effector SAP11 impacts the development of the host plant and enhances its capacity to support reproduction of the insect vector through modulation of the jasmonate pathway (Sugio et al., 2011). SAP11 and its homologs were found to interact with specific transcription factors of the host (Sugio et al., 2011; Janik et al., 2017; Chang et al., 2018; Wang et al., 2018c; Pecher et al., 2019). Other reports indicated that SAP11 was also involved in interfering with the immune system and metabolic responses of the host plant ( $\mathrm{Lu}$ et al., 2014b; Tan et al., 2016). Importantly, immunolocalization experiments showed that Tengu and SAP11 were found in plant tissues other than the phloem sieve tubes where phytoplasmas are confined, confirming that these effectors are secreted by phytoplasmas and then taken up by host sink tissues (Bai et al., 2009; Hoshi et al., 2009). SAP54 and PHYL1 are two homologous phytoplasma effectors that induce spectacular transformations of floral parts into leaf-like organs through destabilization of MADS-box transcription factors of the host (MacLean et al., 2011, 2014; Maejima et al., 2014, 2015; Kitazawa et al., 2017). For simplicity, in the following the SAP54/PHYL1 genes will be referred to as SAP54. Detailed molecular structures for SAP54 and its homologs are now available (Iwabuchi et al., 2019; Liao et al., 2019). Amazingly, it seems that a major role for SAP54 is to promote the attraction of insect vectors by the host plant (Orlovskis and Hogenhout, 2016). Phytoplasma effectors other than Tengu, SAP11, SAP54 have also been shown to interfere with the immune system of the host plant. Notably, it was established that part of the coding sequence of PM19_00185 from 'Candidatus ( $\mathrm{Ca}$.) Phytoplasma mali' induced susceptibility of Arabidopsis thaliana to Pseudomonas syringae pv tabaci, likely through a E3 ubiquitin ligase activity (Strohmayer et al., 2019). The SWP11 gene product from wheat blue dwarf phytoplasma induced cell death in Nicotiana benthamiana, and strikingly, the SWP12 and SWP21 (Tengu) proteins were able to counteract this process (Wang et al., 2018b). As effectors appear to be major players in the interaction with the host plant, their identification through genome mining stands as a prerequisite for a better understanding of phytoplasma diseases.

Identification of phytoplasma effectors only through sequence similarity is unlikely to succeed as they do not seem to be shared with other pathogens (Bai et al., 2009). However, the requirement to cross the plasma membrane to interact with host components leads to the detectable presence of features associated with secretion. A single secretion system is presumed to operate in phytoplasma cells. The initial finding of genes encoding essential components of the Sec translocation system in ' $\mathrm{Ca}$. P. asteris' strain OY supported the existence of a functional Sec system in phytoplasmas (Kakizawa et al., 2001). This evidence was consistent with the cleavage of a N-terminal signal peptide observed for the antigenic membrane protein Amp (Barbara et al., 2002; Kakizawa et al., 2004). The analysis of other phytoplasma genomes indeed confirmed that they encode a Sec-dependent secretion system (Hogenhout et al., 2008; Kube et al., 2012). In Escherichia coli, the SecY, SecE, and SecG gene products assemble into a membrane-integrated protein conducting channel (Tsirigotaki et al., 2017). The $\sec G$ gene appears to be absent in phytoplasmas (Kube et al., 2012), but it may be dispensable (Akimaru et al., 1991; Kube et al., 2014). From studies in E. coli, two pathways guide the Sec substrates to the membrane pore (Cranford-Smith and Huber, 2018). In the first one, a so-called signal recognition particle encoded by the ffh gene binds to the N-terminal sequence constituting the signal peptide of the nascent translation product emerging from the ribosome. It then docks to the FtsY protein, which ultimately allows the direct transfer of the polypeptide to the translocon. The ribosome provides the driving force that feeds the elongating polypeptide into the pore. The presence of conserved fts $Y$ and ffh genes in genome sequences strongly suggest that this pathway also occurs in phytoplasmas (Kube et al., 2012). The second pathway is uncoupled from translation by ribosomes. In that case, chaperones such as trigger factor and $\mathrm{SecB}$ bind to the synthesized polypeptides and keep them in an unfolded state. The substrates are routed to or bound by SecA, which then acts as an ATP-dependent motor that pumps the substrate into the translocon. The SecA gene product was detected in multiple phytoplasmas (Wei et al., 2004). Unlike secA and trigger factor, the $\sec B$ gene is not found in phytoplasma genomes, but other chaperones like GroEL/GroES or DnaK and DnaJ may functionally replace it, based on studies in E. coli (Siewert et al., 2014a). This second pathway is therefore also very likely to be functional in phytoplasmas. It is thought that the translationuncoupled pathway allows to speed up the translocation process, as it is not limited by the ribosome elongation speed (CranfordSmith and Huber, 2018). The pathway taken by each substrate depends upon the hydrophobicity of its N-terminal signal sequence (Tsirigotaki et al., 2017).

The signal sequences that allow recognition of the substrates to be translocated by the Sec machinery are typically located at the $\mathrm{N}$-terminal end of the polypeptide. They are variable in sequence but share a common structure composed of a positively charged stretch of residues ( $n$-region), a hydrophobic core ( $h$-region) and a polar C-terminal domain containing the cleavage site (c-region) (Natale et al., 2008). The interaction with the Sec machinery elements is realized at the level of the $n$ - and $h$-region, and modification of the charge, length, hydrophobic density or alphahelix propensity of the different domains can impact the secretion outcome (Adams et al., 2002; Natale et al., 2008; Freudl, 2018). The presence of a hydrophobic region within signal peptides explains why they can be confused with transmembrane regions by prediction software, and vice-versa. Indeed, signal peptides can even be converted into $\mathrm{N}$-terminal transmembrane regions by increasing the length of the h-region (Nielsen et al., 2019). The link between signal peptides and transmembrane domains is further reinforced by the fact that the translocation activity of the Sec machinery is required for correct insertion of membrane proteins into the plasma membrane. Indeed a lateral gate in the membrane pore allows translocating transmembrane domains to be released (Kuhn et al., 2017). The YidC insertase, also found 
in phytoplasmas, may assist the Sec machinery for this task, or work alone in the same purpose (Kuhn et al., 2017). After the translocation, the signal peptide is cleaved by a signal peptidase and then degraded by a signal peptide peptidase (Kim et al., 2008; Saito et al., 2011). Two types of signal peptidase have been described. Type I signal peptidase recognizes an A-X-A consensus sequence in its substrates and cleaves off the signal peptide (Paetzel, 2014), whereas type II signal peptidase catalyzes the cleavage of diacyl-glycerol-modified substrates, leading to the release of lipoproteins (Narita and Tokuda, 2017). The type II pathway seems to be missing in phytoplasmas, as discussed in the present work.

The identification of putative effectors from phytoplasma genomes thus relies on the detection of signal peptides and subsequent evaluation of the obtained hits. Different software packages among the most popular and efficient ones have been used in previous studies: SignalP 3.0 HMM (Bai et al., 2009; Saccardo et al., 2012; Chen et al., 2014; Music et al., 2018; Wang et al., 2018a), SOSUI and SignalP (Hoshi et al., 2009), SignalP 4.0 (Chung et al., 2013), SignalP 3.0 and 4.1 (Anabestani et al., 2017), SignalP 5.0 (Cho et al., 2019, 2020), Phobius (Kube et al., 2012, 2014; Siewert et al., 2014b; Quaglino et al., 2015), Phobius and SignalP 3.0 HMM (Sparks et al., 2018). SignalP in its first version was available online almost 25 years ago (Nielsen et al., 1997) and has been regularly updated and improved. SignalP 2.0 featured a hidden Markov model (HMM) along with artificial neural networks. SignalP 3.0 was released in 2004 and showed improved performance due to correction of errors in training sets, modification of the neural network design, and creation of a new score to discriminate between signal peptide and non-signal peptide sequences (Bendtsen et al., 2004). In SignalP 4.0 the HMM part was removed, and two neural networks were made available, including one that was trained with transmembrane sequences as negative data to improve the discrimination between signal peptides and transmembrane domains (Petersen et al., 2011). SignalP 4.0 was updated to 4.1 when a greater choice of options was allowed (Nielsen, 2017). Compared to SignalP version 4, SignalP 5.0 benefits from an internal algorithm more suited to signal peptides, a modified output score, and training on a dataset grouping (rather than separating) sequences from Eukarya, Gram-negative bacteria, Gram-positive bacteria, and Archaea (Almagro Armenteros et al., 2019). SignalP 5.0 also simultaneously differentiates Sec and Tat (twin-arginine translocation pathway, unidentified in phytoplasmas so far) substrates without having to rely on specialized separated software. Benchmark tests indicated that incremental versions of SignalP showed superior performance to previous versions, with the notable exception that SignalP 5.0 ranked second after SignalP 4.1 regarding the prediction of type I signal peptides (i.e., cleaved by type I signal peptidase) in Gram-positive bacteria (Almagro Armenteros et al., 2019). Phobius was released in 2004 and is based on a HMM. It was designed with an emphasis on the separation of transmembrane domains and signal peptides, and is available online (Käll et al., 2004, 2007). An important point is that the training datasets of SignalP 4.0 (and presumably of other versions) did not include sequences from Mycoplasma and related genera (Petersen et al.,
2011). On the other hand, Bai et al. (2009) showed that SignalP 3.0 efficiently detected a signal peptide in a set of Mollicutes proteins. It therefore appeared of special interest to determine how these software products perform on phytoplasma sequences.

In this work, we compared the prediction results of these signal peptide predictors, in order to enable informed decisions when mining phytoplasma genome sequences for candidate effectors. We restricted our study to software and configurations already used in previous reports about phytoplasma effector analyses (i.e., SignalP versions 3.0, 4.0, 4.1, 5.0, and Phobius), with the aim to provide a reasonably thorough presentation of the results, and included the TMHMM software for comparison. We examined prediction results for all publicly available phytoplasma sequences that code for the documented effectors Tengu, SAP11, and SAP54 and homologs, for substrate-binding proteins, putative effectors, and specific membrane proteins.

\section{MATERIALS AND METHODS}

Identification of the phytoplasma homologous sequences was performed using the BLASTP software (Camacho et al., 2009) against the 'non-redundant' database (NCBI Resource Coordinators, 2018) with default parameters at the NCBI website. For the SAP54 dataset, the sequences from the phyl-B group of Iwabuchi et al. (2020) were excluded as they did not show the phyllody inducing phenotype observed with other members, although they may still have a functional signal peptide and yetto-discover functions. For Amp and Imp, that can be highly variable, we first extracted from draft or complete phytoplasma genomes the coding sequences located between groEL and nadE, and DnaD and PyrG respectively. We then used the translated sequences as BLASTP queries to retrieve the full dataset of Amp and Imp homologous sequences. To ensure that our dataset was as exhaustive as possible, a keyword search ("antigenic membrane protein phytoplasma" and "imp" respectively) was also performed at Genbank, and validated hits from both strategies were merged.

For all datasets, sequences that were obviously truncated in $\mathrm{N}$ or C-terminal, or exceeding the expected size, were removed from the final sets of hits. Sequence alignments were built with either Muscle 3.8.31 (Edgar, 2004) or Clustal Omega 1.1.0 (Sievers et al., 2011). Alignments were visualized and formatted with Seaview 5.0.4 (Gouy et al., 2010) and Jalview 2.11.1.0 (Waterhouse et al., 2009). Pred-Lipo (Bagos et al., 2008) and LipoP 1.0 (Juncker et al., 2003) were queried online at http://bioinformatics.biol. uoa.gr/PRED-LIPO and http://www.cbs.dtu.dk/services/LipoP respectively. The eulerr package 6.0.0 (Larsson, 2020) was used to draw initial Euler diagrams that were then adapted. Color schemes used in Figures 2, 4 have been elaborated by Paul Tol ${ }^{1}$.

The parameters used for the various versions of SignalP were set following common practice reported for phytoplasma sequences. SignalP 3.0 (Nielsen and Krogh, 1998; Bendtsen et al., 2004) was configured as described in Bai et al. (2009). Thus, in the present study, "SignalP3HMM" refers to the use of

\footnotetext{
${ }^{1}$ https://personal.sron.nl/ pault/
} 
the online version of SignalP 3.0 at https://services.healthtech. dtu.dk/service.php?SignalP-3.0, using the following parameters: organism group: "Gram-positive bacteria"; method: "Hidden Markov Models," Truncation: "Truncate each sequence to max. 70 residues" (default). Predictions with a Sprob score greater than or equal to 0.5 (default) were considered as signal peptide predictions, independently of the position of the predicted cleavage site. The neural network of SignalP 3.0 was not used. SignalP 4.0 (Petersen et al., 2011) was run through the online version at http://www.cbs.dtu.dk/services/SignalP-4.0/. In this study, "SignalP4.0" refers to SignalP 4.0 configured with the parameters indicated by Chung et al. (2013): organism group: "Gram-positive bacteria"; method: "Input sequences do not include TM regions". Only proteins that were indicated as putatively secreted ("?" column) based on the default threshold of 0.57 for $\mathrm{D}$ score were considered to have a positive prediction of signal peptide. Similarly, "SignalP4.1sensitive" refers to the SignalP 4.1 software run online ${ }^{2}$ with parameters indicated in Anabestani et al. (2017): organism group: "Grampositive bacteria"; D-cutoff values: "sensitive," method: "Input sequences do not include TM regions." According to the manual, SignalP 4.1 is the same package as SignalP 4.0 except that some formatting options were added. The "sensitive" option adjusts the $D$-cutoff value at 0.42 instead of 0.57 . The predicted locations of signal peptide cleavage site were taken from the second "pos" column (Ymax score). "SignalP5" designates SignalP 5.0 (Almagro Armenteros et al., 2019) run online at https://services.healthtech.dtu.dk/service.php?SignalP5.0 with the organism group set as "Gram-positive." The TMHMM v2.0 software (noted as "TMHMM" in this study) (Krogh et al., 2001) was used locally or online at https://services. healthtech.dtu.dk/service.php?TMHMM-2.0, leaving the option "Use old model (version 1)" unchecked. Phobius (Käll et al., 2004, 2007) was run online at http://phobius.sbc.su.se/. Phobius can predict the presence of either a signal peptide or a transmembrane domain in protein sequences. This dual output was exploited separately. "Phobius_SP" indicates that only the signal peptide prediction (positive or negative) was considered. For "Phobius_SP_TM," the prediction was taken as positive if either a signal peptide was predicted, as in Phobius_SP, or a transmembrane segment was predicted within the first 50 amino acids of the sequence.

Signal peptide groups used for redundancy removal in prediction counts were established based on signal peptide cleavage sites as predicted by SignalP 4.1. We took care to check that the various predictions were identical for identical signal peptides, and only very few exceptions were found: BAD04276.1 and WP_069028310.1 from the DppA family for SignalP3HMM predictions (data not shown), and the accessions mentioned in Figure 5. In such cases, the sequences were split into two subgroups and each subgroup was then considered as a group.

To build the phytoplasma gene families, the get_homologues software was used (Contreras-Moreira and Vinuesa, 2013). Phytoplasma genomes downloaded from Genbank were provided

${ }^{2}$ https://services.healthtech.dtu.dk/service.php?SignalP-4.1 as input to get_homolog using the "-G -t 0" options and leaving all other options as default.

\section{RESULTS AND DISCUSSION}

\section{Predictions for Tengu, SAP11, and SAP54 Homologs}

Up to now only the Tengu and SAP11 phytoplasma effectors have been demonstrated to be translocated and released extracellularly (Bai et al., 2009; Hoshi et al., 2009). In order to confidently increase the number of signal peptide sequences that could be used to assess the efficiency of signal peptide predictors on phytoplasma sequences, we took advantage of the availability of putative homologs and detailed functional studies. For each of the Tengu, SAP11, and SAP54 effectors, we collected respectively 7,24 , and 25 putative homologous sequences using the BLASTP software (Figure 1 and Supplementary Material M1). Because some of these sequences are shared among several phytoplasma strains, they derive from higher numbers of strains: respectively 22, 33, and 47 strains (Supplementary Tables 1-3). All effector homologs display a putative signal peptide with the expected features, i.e., a positively charged $n$-region, followed by a hydrophobic core and a $c$-region that contains the cleavage site. Interestingly, a large proportion of the mature protein sequences of these homologs have already been subjected to some functional tests validating their activity, for instance phenotyping after in planta expression or impact on expected targets: 6/7 (85.7\%) for Tengu; 13/24 (54.2\%) for SAP11; 14/25 (56\%) for SAP54 homologs (Tables 1-3). This is due to the increasing number of publications devoted to the characterization of phytoplasma effectors, notably regarding their sequence diversity (Sugawara et al., 2013; Maejima et al., 2014; Chang et al., 2018; Iwabuchi et al., 2020; see a complete list of references in Supplementary Tables 1-3).

Based on sequence similarity and functional evidence, we hypothesized that these homologous sequences contain a functional signal peptide. These sequence sets thus constitute a benchmark to evaluate the performance of signal peptide predictors on phytoplasma sequences. However, although there were no duplicates among our homologous sequences $(7+24+25=56$ sequences in total), some had identical signal peptides ("signal peptide sequence group" in Tables 13). We took care to count only non-redundant signal peptide sequences when summarizing the various predictions in Table 4 (33 unique signal peptide sequences in total for accessions associated with functional data, or 43 for all homologs). Accessions with identical signal peptides were checked to have identical predictions. We compared the various flavors of the SignalP software used in phytoplasma effector studies (SignalP versions 3.0, 4.0, 4.1, and 5.0), as well as Phobius (signal peptide prediction referred to as "Phobius_SP" in this study). For SignalP version 4 we collected predictions using either the default thresholds (mentioned as "SignalP4.0"), or the 'Sensitive' 4.1 cut-off values (see section "Materials and Methods"). Given that both signal peptide and transmembrane segments share the presence of a hydrophobic core, predictions performed 

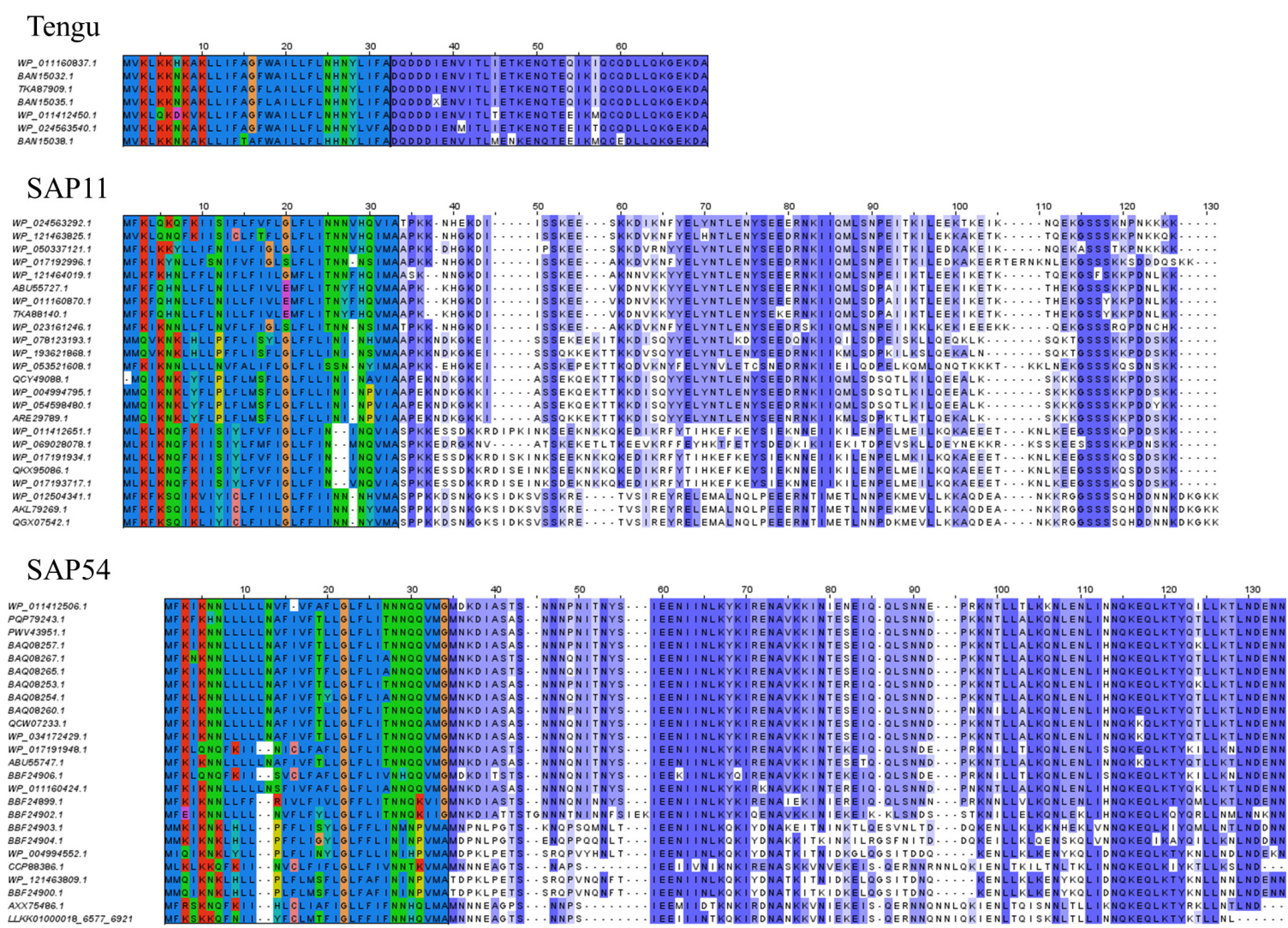

FIGURE 1 | Sequence alignments of the Tengu, SAP11, and SAP54 homologs. The signal peptide regions have been colored according to the biochemical properties of amino acids (red: positively charged residues, blue: hydrophobic residues, green: polar residues, dark pink: negatively charged residues). Mature protein residues have been colored according to amino acid conservation among sequences (violet shades).

by the TMHMM software package (devoted to detection of transmembrane segments) were also included, as well as the cumulated predictions of signal peptide and transmembrane domain by Phobius (see section "Materials and Methods"; indicated by "Phobius_SP_TM" in the text). In both cases, prediction of a transmembrane domain in the $50 \mathrm{~N}$-terminal amino acids was interpreted as a possible signal peptide.

The prediction results for effector homologs with functional evidence readily point to some potential shortcomings for specific predictors (Table 4). Indeed, not all of the sequences were predicted to contain a functional signal peptide by all predictors. The performance of the various predictors differed depending on the homologous sequence set, revealing sequencedependent sensitivity. For the Tengu dataset, the accession WP_011412450.1 that was not detected by SignalP3HMM and SignalP4.0 is present notably in the AYWB (aster yellows witches' broom) phytoplasma genome (Table $\mathbf{1}$ and Supplementary Table 1). Consistently, this sequence was not listed as a putative effector in the original publication on AYWB effectors (Bai et al., 2009), which relied on SignalP 3.0 HMM. Available evidence suggest that the corresponding mature protein is active in planta (Sugawara et al., 2013). In the SAP11 dataset, SignalP3HMM also did not detect a putative signal peptide for three related sequences, unlike all other predictors. The associated mature protein from onion yellows strain OY-M was demonstrated to induce a bushy phenotype and to destabilize expected targets (Chang et al., 2018). Regarding the SAP54

TABLE 1 | Tengu homologs used in this study.

\begin{tabular}{lccc}
\hline Tengu homologs & $\begin{array}{c}\text { SP predicted by all } \\
\text { software }\end{array}$ & $\begin{array}{c}\text { Signal peptide } \\
\text { sequence group }\end{array}$ & $\begin{array}{c}\text { Any functional test } \\
\text { performed }\end{array}$ \\
\hline WP_011160837.1 & $Y$ & 1 & $\mathrm{Y}$ \\
BAN15032.1 & $\mathrm{Y}$ & 2 & $\mathrm{Y}^{*}$ \\
TKA87909.1 & $\mathrm{Y}$ & 3 & $\mathrm{Y}^{*}$ \\
BAN15035.1 & $\mathrm{Y}$ & 3 & - \\
WP_011412450.1 & $\mathrm{N}$ (SignalP3HMM, & 4 & $\mathrm{Y}$ \\
& SignalP4.0) & & $\mathrm{Y}$ \\
WP_024563540.1 & $\mathrm{Y}$ & 5 & $\mathrm{Y}$ \\
BAN15038.1 & $\mathrm{Y}$ & 6 &
\end{tabular}

The second column indicates if a signal peptide is predicted by all of the SignalP3HMM, SignalP4, SignalP4.1sensitive, Phobius_SP, Phobius_SP_TM software (mention " $Y$ " = yes). If not, cells were highlighted in gray (for better readability) with a " $N$ " (no), and predictors that did not produce a positive prediction are mentioned. The third column shows the signal peptide group based on predictions of SignalP4.1sensitive (identical numbers are attributed to identical sequences). Finally, the last column mentions the presence of available evidence of effector activity (any functional test) from literature with the mention " $Y$ "; * cases where the sequence of the mature protein is identical to another accession for which there is available data (see Supplementary Tables 1-3 for references). 
TABLE 2 | SAP11 homologs used in this study.

\begin{tabular}{|c|c|c|c|}
\hline SAP11 homologs & $\begin{array}{c}\text { SP predicted } \\
\text { by all } \\
\text { software }\end{array}$ & $\begin{array}{l}\text { Signal peptide } \\
\text { sequence } \\
\text { group }\end{array}$ & $\begin{array}{c}\text { Any functional } \\
\text { test } \\
\text { performed }\end{array}$ \\
\hline WP_024563292.1 & Y & 1 & Y \\
\hline WP_121463825.1 & Y & 2 & - \\
\hline WP_050337121.1 & Y & 3 & - \\
\hline WP_017192996.1 & Y & 4 & Y \\
\hline WP_121464019.1 & N (Phobius_SP) & 5 & - \\
\hline ABU55727.1 & $\begin{array}{c}\mathrm{N} \\
\text { (SignalP3HMM) }\end{array}$ & 6 & - \\
\hline WP_011160870.1 & $\begin{array}{c}\mathrm{N} \\
\text { (SignalP3HMM) }\end{array}$ & 6 & Y \\
\hline TKA88140.1 & $\begin{array}{c}\mathrm{N} \\
\text { (SignalP3HMM) }\end{array}$ & 6 & - \\
\hline WP_023161246.1 & Y & 7 & Y \\
\hline WP_078123193.1 & Y & 8 & Y \\
\hline WP_193621868.1 & Y & 9 & - \\
\hline WP_053521608.1 & Y & 10 & Y \\
\hline QCY49088.1 & Y & 11 & $Y^{*}$ \\
\hline WP_004994795.1 & Y & 12 & Y \\
\hline WP_054598480.1 & Y & 12 & - \\
\hline ARE29789.1 & Y & 12 & - \\
\hline WP_011412651.1 & Y & 13 & Y \\
\hline WP_069028078.1 & Y & 14 & Y \\
\hline WP_017191934.1 & Y & 13 & - \\
\hline QKX95086.1 & Y & 15 & - \\
\hline WP_017193717.1 & Y & 15 & Y \\
\hline WP_012504341.1 & Y & 16 & Y \\
\hline AKL79269.1 & Y & 17 & $Y^{*}$ \\
\hline QGX07542.1 & Y & 17 & - \\
\hline
\end{tabular}

Column content is described in the caption of Table 1.

dataset, Phobius_SP predicted a signal peptide in only 11 out of 14 sequences. However, for the remaining three sequences, that originate from AYWB phytoplasma, Japanese hydrangea phyllody phytoplasma, and Vc33 phytoplasma, Phobius predicted a N-terminal transmembrane domain instead of a signal peptide. This suggests that transmembrane segment identification could provide, in some cases, useful indication of putative signal peptides. This is independently confirmed by the fact that TMHMM identified a putative transmembrane segment for all sequences of the datasets.

If homologs without functional evidence are included in the analysis, further cases of discrepancies between predictors are revealed. Phobius_SP detected a signal peptide for $86 \%$ of unique N-terminal sequences, followed by SignalP3HMM (93\%), SignalP4.0 and SignalP5 (98\%) (Table 4). Predictions from SignalP4.1sensitive, TMHMM, Phobius_SP_TM suggested the presence of a signal peptide in all (100\%) of the sequences. Only two accessions were predicted to be devoid of a signal peptide by at least two predictors: WP_011412450.1 (Tengu dataset) and BBF24902.1 (SAP54 dataset). A common theme between these two sequences is the presence of a negatively charged residue in the n-region of the putative signal peptide (Figure 1). The three sequences from the SAP11 dataset with a
TABLE 3 | SAP54 homologs used in this study.

\begin{tabular}{|c|c|c|c|}
\hline SAP54 homologs & $\begin{array}{l}\text { SP predicted } \\
\text { by all } \\
\text { software }\end{array}$ & $\begin{array}{c}\text { Signal peptide } \\
\text { sequence } \\
\text { group }\end{array}$ & $\begin{array}{c}\text { Any functional } \\
\text { test } \\
\text { performed }\end{array}$ \\
\hline WP_011412506.1 & N (Phobius_SP) & 1 & Y \\
\hline PQP79243.1 & Y & 2 & Y \\
\hline PWV43951.1 & Y & 3 & $Y^{*}$ \\
\hline BAQ08257.1 & Y & 4 & Y \\
\hline BAQ08267.1 & Y & 5 & $Y^{*}$ \\
\hline BAQ08265.1 & Y & 6 & Y \\
\hline BAQ08253.1 & Y & 4 & - \\
\hline BAQ08254.1 & N (Phobius_SP) & 7 & - \\
\hline BAQ08260.1 & Y & 4 & - \\
\hline QCW07233.1 & Y & 8 & - \\
\hline WP_034172429.1 & Y & 4 & - \\
\hline WP_017191948.1 & Y & 9 & - \\
\hline ABU55747.1 & Y & 4 & - \\
\hline BBF24906.1 & Y & 10 & Y \\
\hline WP_011160424.1 & Y & 11 & Y \\
\hline BBF24899.1 & N (Phobius_SP) & 12 & Y \\
\hline BBF24902.1 & $\begin{array}{c}\text { N } \\
\text { (SignalP3HMM, } \\
\text { SignalP5, } \\
\text { Phobius_SP) }\end{array}$ & 13 & - \\
\hline BBF24903.1 & Y & 14 & Y \\
\hline BBF24904.1 & Y & 15 & - \\
\hline WP_004994552.1 & Y & 16 & Y \\
\hline CCP88386.1 & Y & 17 & Y \\
\hline WP_121463809.1 & Y & 18 & - \\
\hline BBF24900.1 & Y & 18 & Y \\
\hline$A X X 75486.1$ & Y & 19 & - \\
\hline LLKK01000018_6577_6921 & N (Phobius_SP) & 20 & Y \\
\hline
\end{tabular}

Column content is described in the caption of Table 1.

negative prediction by SignalP3HMM also showed a negatively charged residue within the $h$-region. Such residues may prompt signal peptide predictors to predict an absence of signal peptide, even though counter-examples can be found, consistently with the global charge of the $n$-region being likely more relevant than the simple presence of negatively charged residues. Another interesting point is the presence of two gene copies encoding SAP11 homologs in the genomes of ' $\mathrm{Ca}$. P. ziziphi' and 'Parthenium hysterophorus' phyllody phytoplasma. In contrast to two periwinkle leaf yellowing phytoplasma strains and one onion yellows strain that also possess two SAP11 copies (Cho et al., 2019), both homologs from each of them possess a putatively functional signal peptide. Finally, it should be emphasized that both SAP11 and SAP54 contain a SVM (Sequence Variable Mosaic) motif (pfam 12113) (Jomantiene et al., 2007). This motif is presumed to encode a signal peptide and seems to be well recognized by signal peptide predictors in general.

\section{Predictions for SBP Genes}

In their seminal paper, Bai et al. (2009) emphasized that the detection of signal peptides in sequences similar to solute-binding proteins (SBP) strengthened the signal peptide predictions. 
TABLE 4 | Count of positive signal peptide predictions of the protein sequences homologous to Tengu, SAP11, and SAP54.

\begin{tabular}{|c|c|c|c|c|c|c|c|c|}
\hline & \multicolumn{4}{|c|}{ Homologs with functional tests } & \multicolumn{4}{|c|}{ All homologs } \\
\hline & Tengu & SAP11 & SAP54 & Total & Tengu & SAP11 & SAP54 & Total \\
\hline Unique signal peptides & 6 & 13 & 14 & 33 (100\%) & 6 & 17 & 20 & $43(100 \%)$ \\
\hline SignalP3HMM & 5 & 12 & 14 & $31(94 \%)$ & 5 & 16 & 19 & $40(93 \%)$ \\
\hline SignalP4.0 & 5 & 13 & 14 & $32(97 \%)$ & 5 & 17 & 20 & $42(98 \%)$ \\
\hline SignalP4.1sensitive & 6 & 13 & 14 & 33 (100\%) & 6 & 17 & 20 & $43(100 \%)$ \\
\hline SignalP5 & 6 & 13 & 14 & $33(100 \%)$ & 6 & 17 & 19 & $42(98 \%)$ \\
\hline TMHMM & 6 & 13 & 14 & $33(100 \%)$ & 6 & 17 & 20 & $43(100 \%)$ \\
\hline Phobius_SP & 6 & 13 & 11 & $30(91 \%)$ & 6 & 16 & 15 & 37 (86\%) \\
\hline Phobius_SP_TM & 6 & 13 & 14 & 33 (100\%) & 6 & 17 & 20 & $43(100 \%)$ \\
\hline
\end{tabular}

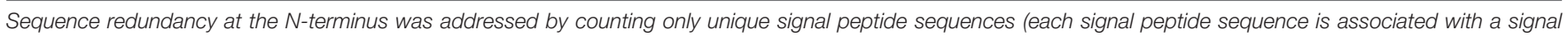
peptide group as indicated in Tables 1-3).

Indeed, SBP are components of ATP-binding cassette (ABC) transporters that bind the substrate to be translocated. In bacteria, SBP are usually located in the periplasm (Gram-negative bacteria) or tethered to the membrane as lipoproteins (Grampositive bacteria) and thus usually possess a signal sequence allowing translocation across the plasma membrane. In order to evaluate the performance of signal peptide predictors on SBP sequence datasets, we used the BLASTP software to identify phytoplasma sequences similar to the AYWB SBPs described in Bai et al. (2006), i.e., NlpA, ArtI, DppA, MalE, ZnuA, PotD. Among them the potD gene products contain a C-terminal transmembrane domain and therefore have a different predicted topology, so we chose to not include them in our study. We could collect at least one sequence from all deposited phytoplasma genomes, thus representing the widest possible phylogenetic diversity, even if some groups are more represented than others (Supplementary Material M1 and Supplementary Table 4). We manually removed sequences that were obviously truncated, and followed the same process as for Tengu, SAP11, SAP54 homologs by counting signal peptide predictions for each of the different predictors after removal of duplicated signal peptide sequences (Table 5).

Only three software packages suggested the presence of a signal peptide for the totality of the 91 sequences: SignalP4.1sensitive, TMHMM, and Phobius_SP_TM. For the other software products, the predictions ranged from $22 \%$

TABLE 5 | Count of positive signal peptide predictions for each SBP family.

\begin{tabular}{lcccccc}
\hline & NIpA & Artl & DppA & MalE & ZnuA & Total \\
\hline Unique signal peptides & 14 & 9 & 30 & 18 & 20 & $91(100 \%)$ \\
SignalP3HMM & 8 & 5 & 16 & 13 & 12 & $54(59 \%)$ \\
SignalP4.0 & 14 & 9 & 28 & 14 & 18 & $83(91 \%)$ \\
SignalP4.1sensitive & 14 & 9 & 30 & 18 & 20 & $91(100 \%)$ \\
SignalP5 & 1 & 0 & 2 & 9 & 10 & $22(24 \%)$ \\
TMHMM & 14 & 9 & 30 & 18 & 20 & $91(100 \%)$ \\
Phobius_SP & 8 & 3 & 2 & 3 & 4 & $20(22 \%)$ \\
Phobius_SP_TM & 14 & 9 & 30 & 18 & 20 & $91(100 \%)$ \\
\hline
\end{tabular}

Like in Table 4, only unique signal peptides are counted.
(Phobius_SP) to 91\% (SignalP4.0). The current latest version of SignalP, SignalP 5.0, detected a signal peptide only in $24 \%$ of these sequences. The sequences that were predicted to have a signal peptide varied depending on the predictor, showing that each predictor has its own specificity (Figure 2).

The SBP sequences were closely examined for the presence of a signal peptide and SBP features. Sequence alignments clearly suggested the presence of typical signal peptides with a short positively charged N-terminus followed by a hydrophobic stretch of amino acids (see Figure 3 for the example of NlpA protein sequences). We found only one group of exceptions where conserved SBP sequences from the NlpA family lacked a signal peptide and were not associated with an ABC-transporter operon (Supplementary Table 5). Each of the corresponding genes was located in tandem with another gene encoding a SBP devoid of a putative signal peptide. These gene products

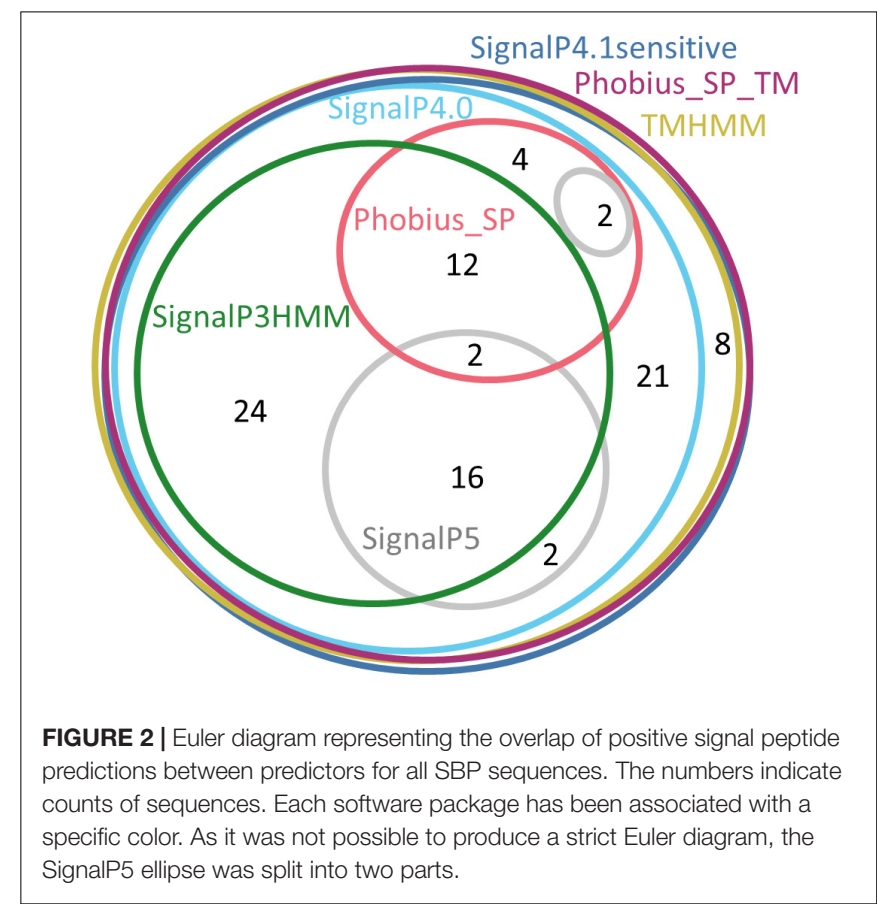




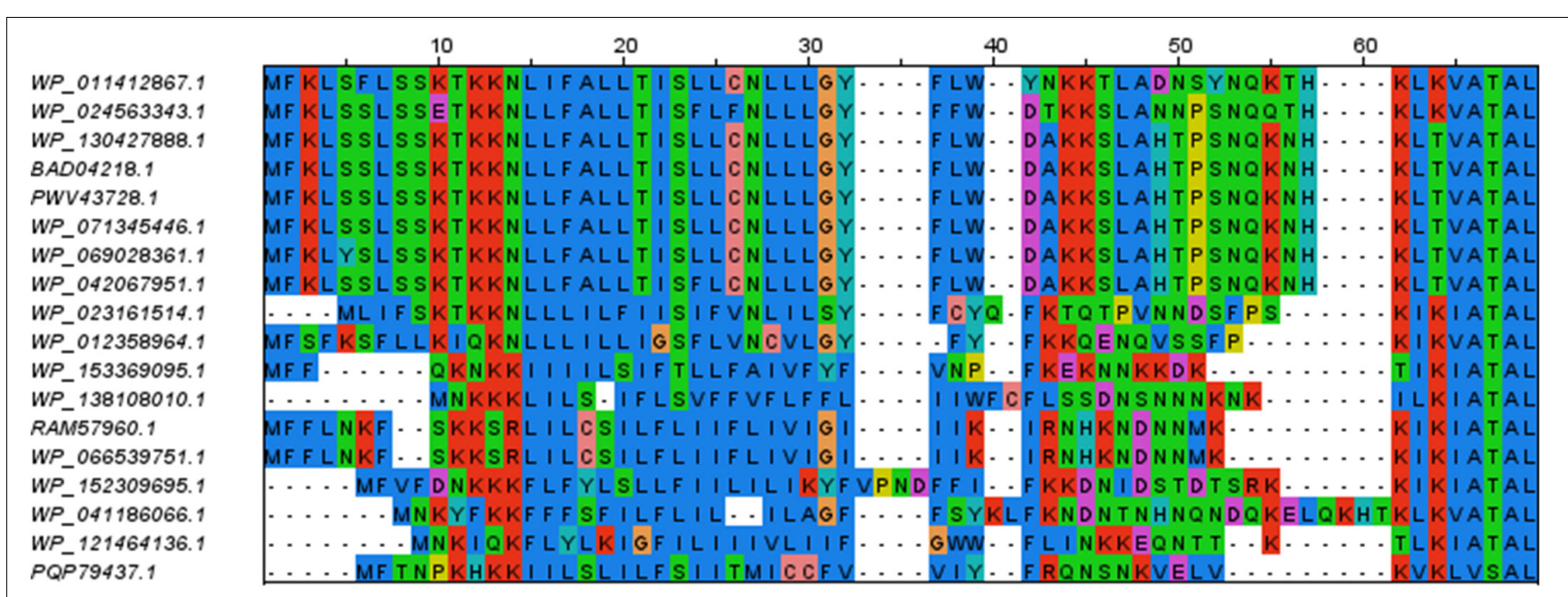

FIGURE 3 | Sequence alignment of the N-terminal residues of members of the NipA family. The remaining of the alignment (C-terminal parts) is not shown. The color code is the same as in Figure 1. Positively charged residues and stretch of hydrophobic residues of the signal peptides are well conserved.

might be involved in intracellular signaling (Scheepers et al., 2016) and were not included in our analyses. We also examined carefully the sequences hits recovered by BLASTP but apparently lacking a complete signal peptide. They were excluded from this study, but were found to result from either one of the following artifacts: (i) N-terminal sequence is not available (i.e., incomplete genomic sequence), (ii) frameshifts disrupted the reading frame, but there is a reading frame encoding the missing part of the signal peptide, (iii) an alternative start codon was used for conceptual translation. In only two cases, we could not succeed in detecting a classical signal peptide (accessions PQP79209.1 and WP_050337100.1 from two different strains of 'Ca. P. phoenicium,' $z$ nuA gene family). In addition, the sequences identified by using BLASTP showed sequences signatures associated with SBP activity (accessions cl21456, cd00995, cl01709, or cl00262 of NCBI Conserved Domain Database). We confirmed that their encoding genes are located at close proximity (presumably within same operon) to other $\mathrm{ABC}$ transporter components, i.e., ATP-binding and permease subunits. Exceptions were $d p p A$ sequences of ' $C a$. P. oryzae,' Cynodon dactylon phytoplasma, and sugarcane grassy shoot phytoplasma, that are isolated but with the corresponding operon being devoid of SBP gene (Supplementary Figure 1 and data not shown). Finally, a few SBP sequences of the DppA family were found to be duplicated or even triplicated within their operon, notably in the 16SrI and 16SrXII groups, explaining the higher number of sequences for this family (Supplementary Table 4). In some cases, such duplications events were associated with loss of typical signal peptide for one gene copy, but in all cases at least one copy with a typical signal peptide remained. The $d p p A$ gene family also showed the highest sequence diversity, as well as differences in the order of the subunits within the $A B C$ transporter operon (Supplementary Figure 1). In summary, sequence analysis supports the presence of a genuine signal peptide in all selected SBP sequences (Supplementary Table 4). Even if no experimental data is available regarding the secretion of these phytoplasma SBPs, the presence of a secretion signal is consistent with the subcellular location required for their activity and what is generally observed in bacteria.

As mentioned above, SBP are usually found as lipoproteins in Gram-positive bacteria (van der Heide and Poolman, 2002). In that case, the preprotein is acylated by Lgt transferase activity on a specific cysteine residue that is part of a motif termed lipobox, and the signal peptide is cleaved by the type II signal peptidase LspA, leaving the modified cysteine residue as the first amino acid of the mature protein (Narita and Tokuda, 2017). However, it is unlikely to be the case for phytoplasmas. Bai et al. (2009) already noted that none of the AYWB $n l p A$, artI, $\operatorname{dppA}$, malE, znuA gene products were predicted as a lipoprotein. We submitted the 115 phytoplasma SBP sequences validated in this study (Supplementary Table 4) to lipoprotein predictors Pred-Lipo and LipoP, and noticed that respectively none and 13 of them were predicted to contain a type II signal peptide (Supplementary Table 6). We believe that the positive type II predictions are artifacts due the presence of a cysteine located close to the cleavage site. The fact that this cysteine is not conserved, even in closely related sequences for some cases, suggests that it is not strictly required (example in Supplementary Figure 2). We think it unlikely that related phytoplasmas with similar SBP protein sequences would resort on different molecular mechanisms for secretion. Huang and Ho (2007) analyzed the $d p p A$ sequence of Loofah witches' broom phytoplasma and suggested the presence of a lipobox. However, its position would imply an unusually long signal peptide of 54 residues and this motif is not conserved among the other phytoplasma $d d p A$ sequences. Another element against the presence of lipoproteins in phytoplasmas is the apparent absence of $\operatorname{lgt}$ and $l s p A$ genes in phytoplasma genomes. We noted with interest that the situation is different for Acholeplasma sp., where most of the identified SBP are predicted to contain a type II signal peptide by Pred-Lipo and LipoP (Supplementary Table 7), and contain a typical lipobox (data not shown). 
In addition, Acholeplasma genomes possess readily detectable $\lg t$ and $l s p A$ genes, and protein acylation was demonstrated in this genus (Serebryakova et al., 2011). These observations raise interesting questions about the evolution of phytoplasmas from acholeplasma-like ancestors and how the transition from lipoprotein SBP to non-lipoprotein SBP occurred.

On a functional point of view, if phytoplasma SBPs are not lipoproteins, how does it happen that they do not diffuse away from phytoplasma cells when their peptide signal is cleaved? A hypothetical scenario could be that the signal peptide is not cleaved, acting as a membrane anchor. An alternative hypothesis with perhaps more experimental grounds is that the SBP remains bound to the ABC transporter subunits, whether it has captured a ligand or not. This has been shown to occur for the histidine transporter of Salmonella typhimurium (Ames et al., 1996).

\section{Predictions for Selected Putative Effector Families}

In order to enlarge further our datasets containing signal peptides, we looked for phytoplasma gene families coding for putative effectors. Selection criteria were based on the number of members, presence within several phytoplasma phylogenetic groups, overall levels of conservation but also sequence variation in the region of the putative signal peptide. These criteria were designed to select for consistent families that allow to challenge signal peptide predictors and discriminate between them. We excluded sequences starting with a SVM motif. Among the few such gene families that were found, we selected three of them that were named after their member from AYWB (respectively AYWB_387, AYWB_376, and AYWB_042). The sequences and list of accessions used in this study are available in Supplementary Material M1 and Supplementary Table 8. Unlike AYWB_042, AYWB_387 and AYWB_376 had already been identified as containing a putative signal peptide and were also designated respectively as SAP08 and SAP09 (Bai et al., 2009).

In a similar process as above, we performed signal peptide predictions for each of the family members and counted the number of putative signal peptides detected by the various software packages (Table 6). Similarly to the Tengu/SAP11/SAP54 effectors and SBP sequences, only

TABLE 6 | Count of positive signal peptide predictions in the AYWB_387, AYWB_376, AYWB_042 families.

\begin{tabular}{lcccc}
\hline & AYWB_387 & AYWB_376 & AYWB_042 & Total \\
\hline Unique signal peptides & 11 & 21 & 12 & $44(100 \%)$ \\
SignalP3HMM & 6 & 21 & 2 & $29(66 \%)$ \\
SignalP4.0 & 11 & 21 & 11 & $43(98 \%)$ \\
SignalP4.1sensitive & 11 & 21 & 12 & $44(100 \%)$ \\
SignalP5 & 5 & 9 & 3 & $17(39 \%)$ \\
TMHMM & 11 & 21 & 12 & $44(100 \%)$ \\
Phobius_SP & 4 & 6 & 1 & $11(25 \%)$ \\
Phobius_SP_TM & 11 & 21 & 12 & $44(100 \%)$ \\
\hline
\end{tabular}

Like in Tables 4, 5, only unique signal peptides are counted.
SignalP4.1sensitive, TMHMM and Phobius_SP_TM predicted that $100 \%(n=44)$ of these sequences included a signal peptide. SignalP4.0 followed closely with 98\%, and SignalP3HMM, SignalP5, and Phobius_SP respectively detected a signal peptide in only 66,39 , and $25 \%$ of the sequences. Again, the sequences that were predicted to be devoid of a signal peptide varied depending on the software (Figure 4). Only eight accessions were predicted to contain a signal peptide by all of the software packages. A major part (8 out of $11-73 \%$ ) of positive predictions by Phobius_SP were also realized by all other software, the remaining (27\%) being in contradiction with SignalP5 predictions. The cumulated positive predictions of SignalP3HMM, SignalP5 and Phobius_SP did not include the totality of the sequences (32 sequences out of $44-73 \%$ ).

A not-yet-solved question is whether the sequences of this dataset do include a functional signal peptide that is processed by the secretion machinery. We currently have no experimental evidence of secretion for any of them, but the conservation of positively charged amino-acids before a core of hydrophobic residues, which are typical features of signal peptides, provides support for this hypothesis (sequence alignments in Supplementary Figures 3-5). Incidentally, in most cases the genes of the AYWB_387 and AYWB_376 families are located close to PMU-associated genes in genomic sequences, with PMU being presumed effector-rich regions (Sugio and Hogenhout, 2012; Chung et al., 2013; Ku et al., 2013). For example, in AYWB genome, the $A Y W B \_387, A Y W B \_376$, and $S A P 11$ genes are included in a region of $11.2 \mathrm{~kb}$ that also features PMU elements (Bai et al., 2009). In some genome drafts, $A Y W B \_387$ and $A Y W B \_376$ homologs are located in short contigs whose ends could not be assembled, likely due to repeated sequences such as PMUs. The duplication of family members in

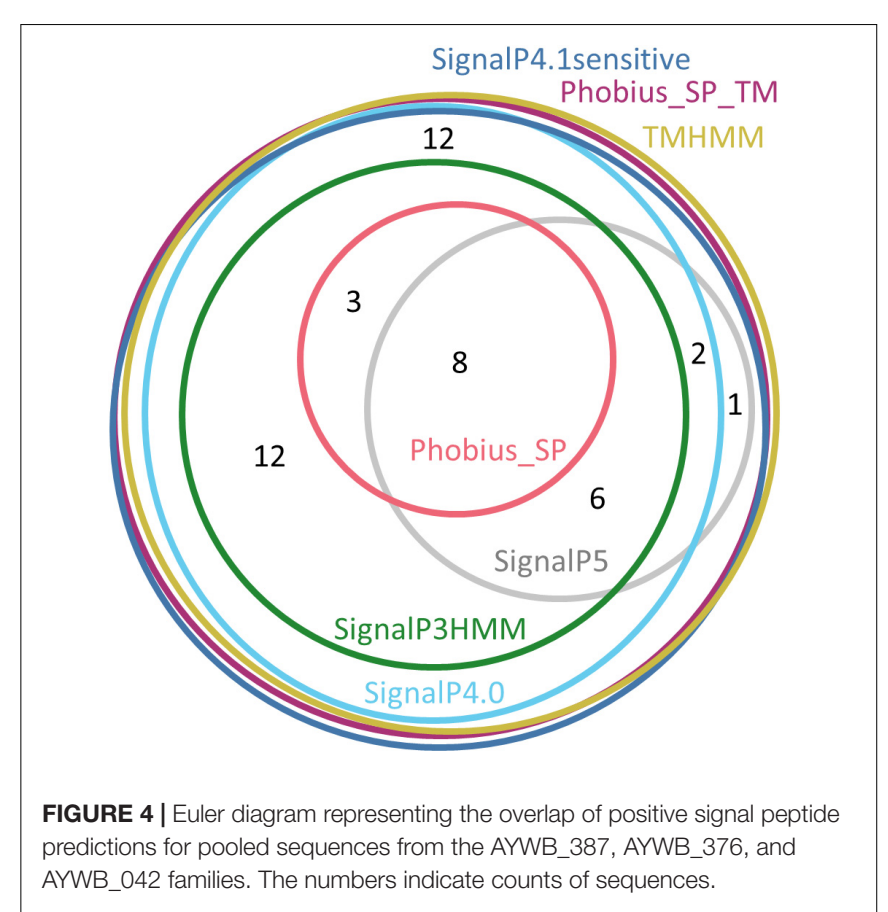


some genomes (Supplementary Table 8) is also linked to their location in PMU regions.

The fact that some, but not all, of the homologs of AYWB_387, AYWB_376, and AYWB_042 were predicted to have a signal peptide depending on software packages, highlights the limits of signal peptide predictions and illustrates the differences between signal peptide predictors. Whatever the true secretion status of these gene products, the most consistent predictors within each family are SignalP4.1sensitive, TMHMM and Phobius_SP_TM. This dataset also provides examples of how predictors can be specifically affected by variations in primary sequence. We noticed that very similar $\mathrm{N}$-terminal sequences sometimes resulted in different prediction outcomes, particularly with SignalP5, as shown in Figure 5. In the AYWB_376 family, the accessions WP_053521373.1 and WP_011412494.1 display a single amino acid variation within a hydrophobic stretch in their first 60 amino acids (leucine to phenylalanine at position 29), yet they have different SignalP5 prediction. A possible explanation could be an interference with cleavage site recognition, as cleavage is predicted at position 30. However, accessions WP_011412494.1 and WP_121463821.1 are identical on their first 57 residues, well over the whole putative signal peptide, but have different SignalP5 predictions, illustrating the high sensitivity of SignalP5. Another example from the same family shows that replacement of one hydrophobic amino acid by another within the hydrophobic core also leads to differing Phobius predictions, in that case transmembrane domain or signal peptide. This example shows how the prediction consistency is increased if $\mathrm{N}$-terminal transmembrane domains are considered as a possible indication of a signal peptide ("Phobius_SP_TM" in Tables 46). In a last example from the ZnuA SBP family, accessions WP_017192577.1, WP_017193516.1, and WP_017193007.1 have identical N-termini for 62 residues but varying signal peptide predictions by SignalP5 (Figure 5). As the putative signal peptide is only 27 amino-acids, as predicted by SignalP5 itself, such a difference of prediction output is surprising. These particular cases suggest that the high sensitivity of SignalP5 resulting from its design and training set may provide excellent performance in general but might not be adapted to phytoplasma sequences.

\section{Predictions for Selected Membrane Proteins}

As increased detection sensitivity can be associated with increased false positive risk, we challenged the evaluated predictors with sequences that resemble signal peptides. In order to collect such sequences, we looked for phytoplasma gene families satisfying several criteria. First, they should be widely conserved among phytoplasmas, allowing to observe a conserved trend and not to rely only on few particular sequences. Second, the sequences from this family should include a hydrophobic stretch of residues in their N-terminus, but, unlike typical signal peptide sequences, only rare positively charged amino acids in the $n$-region. Thus, in accordance with data from other studies (see below) and the positive-inside rule (Baker et al., 2017), these proteins presumably contain a N-terminal transmembrane domain that leaves the bulk of the protein in the cytosol, except a very short extracellular domain. As a consequence, these sequences can be used to evaluate the ability of signal peptide predictors to discriminate typical signal peptides and transmembrane domains. The accessions and sequence alignments of these four families are available in Supplementary Tables 9,10 and Supplementary Figures 6-9).

The first selected gene family encode RmuC, of which the precise function is unknown, but that has been associated with DNA recombination processes (Slupska et al., 2000). Intriguingly, the RmuC gene was detected only in $16 \mathrm{SrI}$ and 16 SrXII phytoplasma groups, as briefly mentioned by Kube

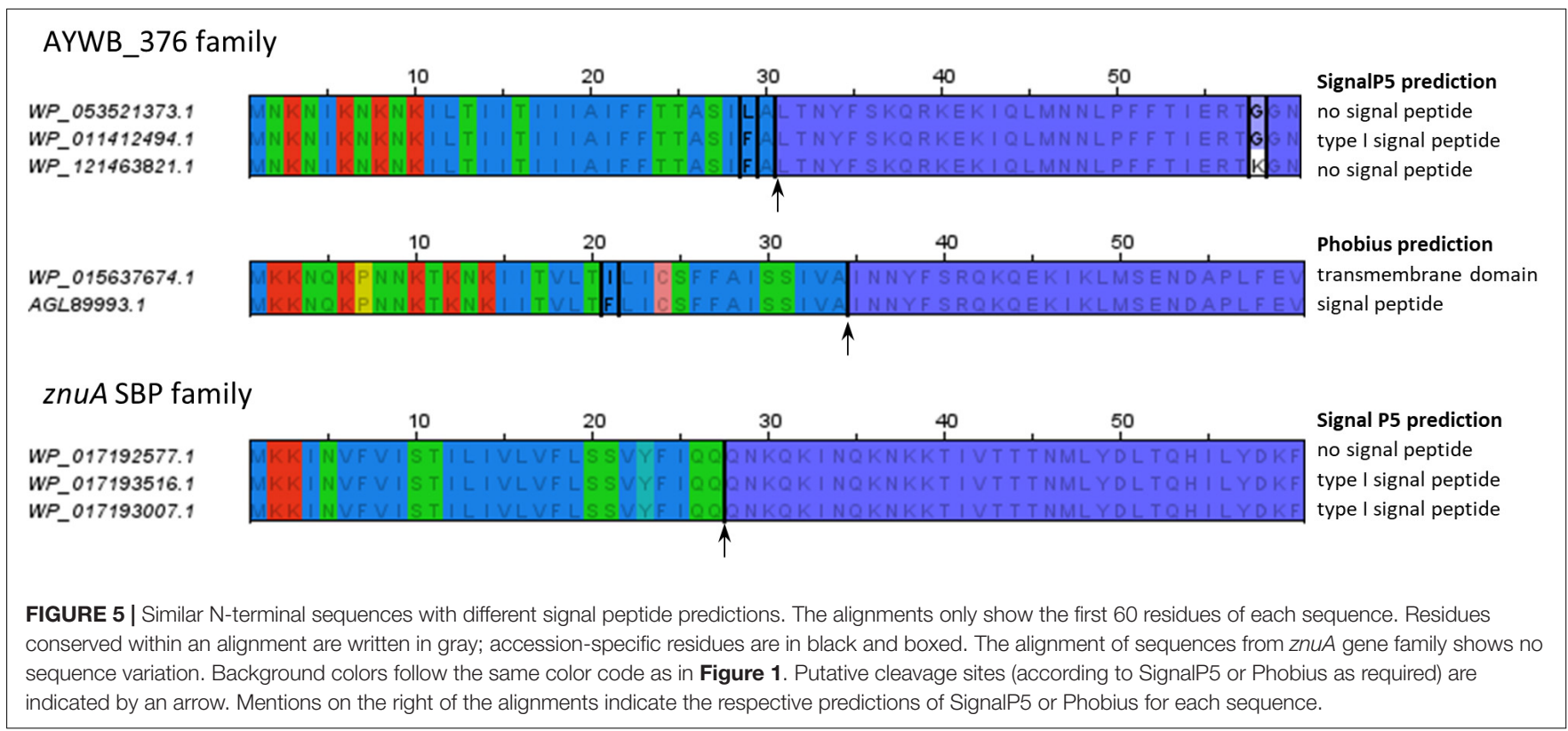


et al. (2012) and Saccardo et al. (2012), whereas it is readily detectable in other genera of the Mollicutes class, such as Acholeplasma and Spiroplasma, as well as many bacterial genera outside Mollicutes. The RmuC protein from E. coli is also predicted to contain a transmembrane domain at its $\mathrm{N}$-terminus (Lomize et al., 2017). The second family focuses on the YneF gene, that codes for a short protein of about 70 amino acids of unknown function (uncharacterized protein family UPF0154, pfam PF03672). As yneF is widely conserved in phytoplasmas, but also in acholeplasmas and mycoplasmas, and in distant genera such as Bacillus that have a different lifestyle, it is likely to perform a conserved function. We had a similar case with the third family, referred to as AYWB_444 family. This family is shared among phytoplasmas but also with acholeplasmas and other bacterial taxons from Tenericutes. While its function is unknown, and no conserved functional domain has been identified, the conservation range suggests that it is involved in housekeeping functions. The last gene family is RNase Y, which has been involved in mRNA decay in Bacillus subtilis and other Gram-positive bacteria, and for which there is experimental evidence of association with the plasma membrane through a N-terminal membrane-spanning domain (Bechhofer and Deutscher, 2019; Hamouche et al., 2020). Similar sequences are found in phytoplasmas and acholeplasmas, and also in many Gram-positive bacterial taxons. We faced an ambiguity for RNase Y sequences from $16 \mathrm{SrI}$ ribosomal group and ' $\mathrm{Ca}$. P. australiense' genomes, due to two alternative start codons generating protein sequences differing by respectively 11 and 18 amino acids (Supplementary Tables 9,10 and Supplementary Figure 9).

As above, predictions for the different signal peptide predictors were collected and summarized in Table 7. Large differences were observed between predictors, with none of the 68 sequences of the RmuC, YneF, AYWB_444, RNaseY families predicted to contain a signal peptide by Phobius_SP, and only $1 \%$ for SignalP5, whereas SignalP4.1sensitive counts reached close to $70 \%$. These differences were similar at the level of individual gene families, and therefore suggestive of the respective tendency of predictors to consider this type of $\mathrm{N}$-terminal transmembrane domains as putative signal peptides. It is important to note that the frequent occurrence of signal peptide prediction for these membrane proteins by SignalP version 4 is linked with the specific configuration used in this study for the 'method' parameter. SignalP version 4 has been designed with an improved capacity to distinguish signal peptides from transmembrane domains (Petersen et al., 2011). When this ability is switched on by using SignalPTM network (method parameter set to "Input sequences may include TM regions"), only 1 sequence (WP_066539764.1) out of 68 was predicted to contain a signal peptide. This particular accession from ' $\mathrm{Ca}$. P. oryzae' was also predicted to contain a signal peptide by SignalP3HMM and SignalP5, likely because of the presence of three lysine residues before the transmembrane domain, but not by Phobius_SP. Taken as a whole, comparison of Table 7 with Tables 4, 5 clearly illustrate that for the tested predictors, a gain in sensitivity was also associated with an increased risk of false positives when challenged with sequences partially similar to signal peptides.

\section{Predictions for Immunodominant Membrane Proteins}

Unlike membrane proteins described above, immunodominant membrane proteins are phytoplasma membrane proteins for which the bulk of the protein is extracellular and only a very short portion is intracellular (Kakizawa et al., 2006), allowing them to interact with host molecules (Konnerth et al., 2016). They are associated with different gene products of various topologies. Among them, Amp was suggested to possess a cleaved signal peptide and a C-terminal transmembrane domain, based on experimental evidence (Barbara et al., 2002; Kakizawa et al., 2004). We therefore gathered available Amp sequences to generate another dataset of signal peptidecontaining phytoplasma proteins. As Amp proteins are notoriously variable, we proceeded both by keyword search and BLASTP similarities to collect protein sequences. Hundred and eight Amp sequences were found in total, with a presence restricted to phytoplasma ribosomal groups 16SrI, 16SrXII, and 16SrXIII (sequences and accessions in

TABLE 7 | Count of positive signal peptide predictions for the RmuC, YneF, AYWB_444, RNaseY gene families.

\begin{tabular}{lccccc}
\hline & RmuC & YneF & AYWB_444 & RNase $\mathbf{~}$ & Total \\
\hline $\begin{array}{l}\text { Unique N-terminal } \\
\text { sequences }\end{array}$ & 7 & 21 & 21 & 19 & $68(100 \%)$ \\
SignalP3HMM & 1 & 1 & 1 & 1 & $4(6 \%)$ \\
SignalP4.0 & 7 & 8 & 2 & 6 & $23(34 \%)$ \\
SignalP4.1sensitive & 7 & 16 & 12 & $9-12$ & $44-47(65-69 \%)$ \\
SignalP5 & 0 & 0 & 0 & 1 & $1(1 \%)$ \\
TMHMM & 7 & 21 & 21 & 19 & $68(100 \%)$ \\
Phobius_SP & 0 & 0 & 0 & 0 & $0(0 \%)$ \\
Phobius_SP_TM & 7 & 21 & 21 & 19 & $68(100 \%)$
\end{tabular}

For RNaseY and total, a range of values is given, because there is some uncertainty on the translation start codon of some genes. Redundant $N$-terminal sequences were counted only once, as in Tables 4-6. Here, these $N$-terminal sequences were defined operationally as the regions identified as possible signal peptides by SignalP4.1sensitive, even if the signal peptide prediction score of these sequences did not reach the threshold value.

TABLE 8 | Count of positive signal peptide predictions for Amp and Imp sequences.

\begin{tabular}{lcc}
\hline & Amp & Imp \\
\hline Unique signal peptides or N-terminal sequences & $22(100 \%)$ & $43(100 \%)$ \\
SignalP3HMM & $22(100 \%)$ & $16(37 \%)$ \\
SignalP4.0 & $22(100 \%)$ & $12(28 \%)$ \\
SignalP4.1sensitive & $22(100 \%)$ & $43(100 \%)$ \\
SignalP5 & $22(100 \%)$ & $0(0 \%)$ \\
TMHMM & $22(100 \%)$ & $43(100 \%)$ \\
Phobius_SP & $14(64 \%)$ & $0(0 \%)$ \\
Phobius_SP_TM & $22(100 \%)$ & $43(100 \%)$
\end{tabular}

Redundant N-terminal sequences were counted only once, as in Tables 4-7. 
Supplementary Material M1 and Supplementary Table 11). Prediction results by the different predictors after elimination of redundant sequences are shown on Table 8. All tested predictors, excepted Phobius_SP, unanimously detected a signal peptide in all Amp N-terminal sequences, thus achieving a prediction rate of $100 \%$. Phobius_SP predicted a signal peptide for $64 \%$ of Amp sequences, while it identified a transmembrane domain in the remaining sequences. If this prediction is also considered as an indication of the presence of a signal peptide, as for Phobius_SP_TM, the prediction rate also reaches $100 \%$. Thus, like for documented effectors and SBP, transmembrane domain predictors TMHMM or Phobius_SP_TM also allow suggestion of a putative signal peptide in all tested Amp sequences. The analysis of the cases predicted by Phobius to have a transmembrane region instead of a signal peptide suggests that replacement of a single amino acid of the presumed cleavage site leads Phobius to predict a transmembrane domain, whereas the various SignalP versions simply indicated another possible cleavage site located nearby. Alternatively, it is possible that increasing the hydrophobicity close to the cleavage site also promotes prediction of a transmembrane segment by Phobius.

Other phytoplasma proteins have been described with a presumed similar topology: VmpA and $\mathrm{VmpB}$ from $16 \mathrm{SrV}$ group (Renaudin et al., 2015; Malembic-Maher et al., 2020), and Vmp1 from 16SrXII group (Cimerman et al., 2009). Available evidence strongly suggests that a signal peptide is cleaved from VmpA in phytoplasma cells (Renaudin et al., 2015). Only few fulllength sequences could be collected by BLASTP for these proteins (Supplementary Material M1 and Supplementary Table 12). The comparison of predictions shows that for VmpA, only SignalP5 and Phobius_SP did not detect the signal peptide, whereas all predictors identified a signal peptide for $\mathrm{VmpB}$ and Vmp1 (Supplementary Table 12).

Another documented phytoplasma immunodominant membrane protein is Imp. Imp is anchored in the plasma membrane by a $\mathrm{N}$-terminal transmembrane helix (Berg et al., 1999; Kakizawa et al., 2009; Neriya et al., 2011; Siampour et al., 2013). As for Amp, Imp sequences were retrieved using two different approaches based on sequence similarity and keyword searches. Hundred and twenty-three Imp sequences (Supplementary Material M1 and Supplementary Table 13), corresponding to 43 unique $\mathrm{N}$-terminal sequences, were collected and found to originate from all branches of the phytoplasma phylogenetic tree. These sequences were submitted to the predictors tested in this study (Table 8). Results were highly contrasted, as SignalP5 and Phobius_SP detected a peptide signal in none of the sequences, SignalP4.1sensitive in all of the sequences, and SignalP3HMM and SignalP4.0 had positive predictions for 37 and $28 \%$ of the sequences respectively. The transmembrane predictors TMHMM and Phobius_SP_TM detected a transmembrane region in $100 \%$ of the sequences, thus constituting obvious cases of false positives if all positive predictions of transmembrane regions are taken as an indication of signal peptides. As the signal peptide predictors tested in this study have been designed to discriminate between transmembrane regions and signal peptides, for the Imp dataset, the best performance will be associated with the lowest number of positive predictions. Following this principle, regarding the Imp dataset, the best performance comes from SignalP5 and Phobius, whereas the worst performance is produced by SignalP4.1sensitive. However, similarly to the case of membrane proteins discussed above, it must be emphasized that another configuration of SignalP version 4 would lead to very different results. Indeed, if the SignalP-TM network is used, instead of the SignalP-noTM network used throughout this study, no Imp sequence is predicted to contain a signal peptide, again pointing to the major effect of this parameter in the configuration of SignalP version 4.

\section{Global Comparison of Signal Peptide Lengths and Scores}

The availability of the various sequence datasets detailed in this study opens up the possibility to outline conserved properties and possible variations of phytoplasma signal peptides. For instance, in their search of AYWB putatively secreted proteins, Bai et al. (2009) focused on candidates with predicted signal peptides longer than 20 amino acids and shorter than 50 amino acids. How does that rule of thumb fit with the Tengu, SAP11, SAP54, SBP and Amp sequence datasets? The analysis of signal peptide length distribution for these datasets shows that, whatever the predictor, a large fraction of predicted signal peptide sequences displays a length between 30 and 35 residues, and that the 20-50 amino acid length range captures the whole diversity of these datasets (Supplementary Figure 10). Incidentally, the same type of analysis performed on various phytoplasma genomes revealed a similar distribution (data not shown). Notable exceptions came from SignalP4.1sensitive and Phobius, which both predicted signal peptides shorter than 20 amino acids, that belong for some of them to likely housekeeping gene products such as ribosomal proteins (data not shown). Thus, dismissing predictions of signal peptides shorter than 20 residues seems currently to be a reasonable assumption.

The various versions of SignalP also provide access to a score which allows the discrimination of signal peptides from non-signal peptides. This score can be interpreted as the confidence in the signal peptide prediction. Supplementary Figure 11 shows a comparison of these scores across the various datasets. For all the SignalP versions there is a global trend to output lower values with transmembrane protein datasets (RmuC, YneF, AYWB_444, RNAseY). However, scores with moderate values can be achieved by sequences from datasets of different status, suggesting that such indicator values may not be helpful for an uncharacterized gene product. Furthermore, the prediction score, as a confidence score, may indicate the relatedness to signal peptide sequences of the software training set, which might not be fully relevant in the case of phytoplasma sequences.

Finally, we also looked if there was any correlation between cleavage score values and the expected status of the sequence (signal peptide or transmembrane region) (Supplementary Figure 12). Like the predictions scores, we observed that cleavage scores were not highly informative, meaning that moderate values could correspond to sequences with either a signal peptide or a transmembrane domain. 


\section{GENERAL DISCUSSION AND CONCLUSION}

A major question addressed in this study is the identification of software packages best adapted to identify signal peptides from phytoplasma sequences. We could rely notably on sequences of documented effectors, SBP and membrane proteins from several gene families, intending to cover as much as possible of the phylogenetic diversity of phytoplasmas, and thus drawing on conserved biological features rather than isolated sequences. A primary conclusion is that there is no ideally performing software among those that were tested, since none of them simultaneously detects all expected signal peptides and ignores all N-terminal transmembrane domains. However, depending on the final purpose, some of them may appear more suited than others. Biologists seeking confidence in predictions, or instead sensitivity to avoid missing interesting candidates, might choose different options. We observed that SignalP 5.0 and Phobius_SP seemed particularly good at discriminating $\mathrm{N}$-terminal transmembrane domains from signal peptides, however they missed many expected signal peptides. While they are both popular and highly cited software, a likely explanation for this failure in our case is the lack of experimentally proven phytoplasma signal peptide sequences that could be used for software training, whatever the efforts spent in software design. From the results we obtained, it seems that the strength of SignalP 5.0 and Phobius_SP lies in discrimination ability and confidence in their positive predictions, even if a more precise evaluation of this confidence would be required through challenges with many more sequences devoid of signal peptides. Moreover, the various software cannot be simply ranked by increasing order of confidence in positive predictions. Indeed, except for SignalP4.1sensitive, TMHMM and Phobius_SP_TM, predictions have only a partial overlap (Figures 2, 4), leaving room for uncertainty in the case of uncharacterized gene products.

Conversely, SignalP4.1sensitive, and obviously signal peptide suggestions derived from TMHMM and Phobius_SP_TM predictions, were confounded by transmembrane domains, but achieved $100 \%$ of detection of putative signal peptides. They were also more consistent in their predictions from putative effectors (Table 6). Such a success rate is appealing, even if it must be kept in mind that only a part of the sequence space of phytoplasma signal peptides has been tested here. These predictors might be more appropriate when attempting to build an exhaustive set of putatively secreted effector candidates from a phytoplasma genome, at the price of a background noise of false-positive proteins. The fact that all expected signal peptides were detected by transmembrane domain predictors also highlights the relatedness between phytoplasma signal peptides and transmembrane helical segments. Tests by the creators of TMHMM indicated that about $60 \%$ of signal peptides from Gram-positive bacteria were recognized as transmembrane domains, compared to $20 \%$ for sequences of eukaryotic and Gram-negative bacterial origins, probably because of the longer $h$-regions of signal peptides from Gram-positive bacteria
(Krogh et al., 2001). Thus, it seems that phytoplasmas also share this feature with their Gram-positive ancestors, i.e., the h-regions of their signal peptides are similarly sized to alpha-helical transmembrane regions. In turn, this can be exploited by using transmembrane predictors such as TMHMM or Phobius_SP_TM to indicate the possible presence of signal peptides. Moreover, this explains why the SignalP4.1sensitive configuration used here (SignalP-noTM network) also detected $100 \%$ of tested signal peptides, as it is based on a neural network not trained with transmembrane sequences as negative data. Comparison with the other neural network available for SignalP 4.1 (SignalP-TM network) that discriminates transmembrane regions confirmed this conclusion (data not shown).

The close proximity between phytoplasma signal peptides and transmembrane domains may appear as a difficulty when looking for soluble proteins that could act as virulence factors. However, even if the current software packages reach their limits here, and data about relative numbers of secreted soluble proteins and membrane proteins are lacking at the moment, close examination of sequences might help in a few cases. Notably, alignment of the sequence of interest with similar sequences from other phytoplasmas could reveal conserved biological features and amino acid composition trends; the occurrence of a barely positive or even negative charge in the region before the hydrophobic stretch could be suggestive of a transmembrane domain rather than a signal peptide, as shown by $\mathrm{RmuC}$ and RNAseY sequences described above. Moreover, a clear distinction between transmembrane region and signal peptide might not be critical or not necessarily required to identify factors involved in interaction with the host. Indeed membrane proteins can also bind to host components, as illustrated by Amp, Imp and VmpA (Suzuki et al., 2006; Galetto et al., 2011; Boonrod et al., 2012; Arricau-Bouvery et al., 2018). Thus, it may be wise to include selected candidates for functional studies even if early predictions suggest a $\mathrm{N}$-terminal transmembrane domain (see for instance Strohmayer et al., 2019). And finally, in the case of membrane proteins one could even speculate that post-translational cleavage by proteases other than signal peptidase could release active peptides, similarly to the Tengu and SAP11 effectors which have already been shown to be subjected to such proteolysis (Sugawara et al., 2013; Lu et al., 2014a).

We envision this study as the starting point of a journey in phytoplasma effector biology, of which next stops include, among others, the elucidation of the core- and pan-effectome of phytoplasmas, the identification of effectors evolutive history, and relationships with PMUs. We hope that these results will contribute to a better understanding of phytoplasmas and help pathologists in their quest for phytoplasma virulence factors.

\section{DATA AVAILABILITY STATEMENT}

The datasets presented in this study can be found in online repositories. The names of the repository/repositories and accession number(s) can be found in the article/ Supplementary Material. 


\section{AUTHOR CONTRIBUTIONS}

LB and XF provided the initial idea. CG performed the analyses and drafted the manuscript. All the authors contributed to the article and approved the submitted version.

\section{FUNDING}

This work was funded by the INRAE Plant Health and Environment (SPE) division grant ID FESTIV, the Conseil Interprofessionel des Vins de Bordeaux (CIVB) grant ID 41044 and the Conseil Régional Nouvelle Aquitaine grant ID ESR2020 FLADO-INNOV.

\section{ACKNOWLEDGMENTS}

We thank Vincent Baby, Paul Dubos, and Patrice Mora for help with bioinformatics tools.

\section{SUPPLEMENTARY MATERIAL}

The Supplementary Material for this article can be found online at: https://www.frontiersin.org/articles/10.3389/fmicb. 2021.661524/full\#supplementary-material

Supplementary Figure 1 | Genomic environments of SBP coding sequences from DppA family. SBPs are colored in pink, permease subunits in blue and green, and ATP-binding subunits in orange. Accessions retained in the final DppA dataset are mentioned above schemes and their corresponding coding sequences marked with an asterisk

Supplementary Figure 2 | Alignment of selected sequences from the DppA family showing sequence similarity but different lipoprotein predictions. WP_017192229.1, WP_017193400.1, WP_053521415.1, WP_017192740.1, WP_017191628.1 originate from Milkweed yellows phytoplasma, Vaccinium witches'-broom phytoplasma, 'Candidatus Phytoplasma pruni', Italian clover phyllody phytoplasma, Poinsettia branch-inducing phytoplasma respectively. The last four sequences are predicted to contain a type II signal peptide by LipoP (cysteine indicated by an arrow), unlike the first sequence. The five proteins share over $79 \%$ of sequence identity between each pair (as calculated by BLASTP) and are expected to act through similar molecular mechanisms, which does not really fit with the hypothesis that the last four could be lipoproteins but not the first one. Residues have been colored according to their biochemical properties up to position 36 of the alignment, and the remaining of the sequences is colored depending on conservation (color codes are the same as Figure 1).

Supplementary Figure 3 | Sequence alignment for the AYWB_387 family. The residues have been colored according to biochemical properties up to position 42 of the alignment, and the remaining of the alignment is colored according to conservation between sequences with violet shades.

Supplementary Figure 4 | Sequence alignment for the AYWB_376 family. The residues have been colored according to biochemical properties up to position 42 of the alignment, and the remaining of the alignment is colored according to conservation between sequences with violet shades.

Supplementary Figure 5 | Sequence alignment for the AYWB_042 family. The residues have been colored according to biochemical properties up to position 65 of the alignment, and the remaining of the alignment is colored according to conservation between sequences with violet shades.

Supplementary Figure 6 | Sequence alignment for the RmuC family. The residues have been colored according to biochemical properties up to position 50 of the alignment, and the remaining of the alignment is colored according to conservation between sequences with violet shades.
Supplementary Figure 7 | Sequence alignment for the YneF family. The residues have been colored according to biochemical properties up to position 50 of the alignment, and the remaining of the alignment is colored according to conservation between sequences with violet shades.

Supplementary Figure 8 | Sequence alignment of the AYWB_444 family. The residues have been colored according to biochemical properties up to position 50 of the alignment, and the remaining of the alignment is colored according to conservation between sequences with violet shades.

Supplementary Figure 9 | Sequence alignment for the RNAseY family. The residues have been colored according to biochemical properties up to position 65 of the alignment, and the remaining of the alignment is colored according to conservation between sequences with violet shades. In this alignment, only sequences collected from the nr database of NCBI at the date of the study are shown (accessions listed in Supplementary Material SM15). This includes some of the alternative start codons variants (for example accessions AOF54820.1 and WP_069028241.1 that derive from the same gene of maize bushy stunt phytoplasma strain M3), but not the alternative start codons variants mentioned in Supplementary Material 16

Supplementary Figure 10 | Distribution of signal peptide length for the Tengu, SAP11, SAP54, SBP, AYWB_387, AYWB_376, AYWB_042 and Amp datasets. Redundancy between sequences was addressed by counting only unique signal peptides predicted by each software package.

Supplementary Figure 11 | Distribution of prediction scores for all the datasets described in this study. In these plots, each sequence in a given dataset is represented by a dot. Orange dots were assigned to sequences with a positive signal peptide prediction. Sequences that were predicted to have no signal peptide are represented by blue dots.

Supplementary Figure 12 | Distribution of cleavage scores for all the datasets described in this study. In these plots, each sequence in a given dataset is represented by a dot. Orange dots were assigned to sequences with a positive signal peptide prediction. Sequences that were predicted to have no signal peptide are represented by blue dots. For SignalP5, sequences that had no predicted signal peptide were assigned a cleavage score of 0 .

Supplementary Table 1 | Accessions, origin, identity groups, and references for sequences homologous to Tengu.

Supplementary Table 2 | Accessions, origin, identity groups, and references for sequences homologous to SAP11.

Supplementary Table 3 | Accessions, origin, identity groups, and references for sequences homologous to SAP54.

Supplementary Table 4 | SBP accessions used in this study.

Supplementary Table 5 | List of sequences associated with NIpA family that are devoid of signal peptides.

Supplementary Table 6 | List of phytoplasma SBP gene products predicted to be a lipoprotein according to LipoP.

Supplementary Table 7 | Lipoprotein predictions of SBPs from some Acholeplasma genomes. This table is adapted from the Supplementary Material 12 of Kube et al. (2014) J. Mol. Microbiol. Biotechnol. 24, 19-36 that recapitulates SBP genes identified in the genomes of Acholeplasma brassicae, A. palmae, and A. laidlawii. Starting from this data, the locus tags were updated using NCBI annotation, and the corresponding protein sequences were submitted to the PredLipo and LipoP servers. The obtained predictions are listed in the columns "PredLipo" and "LipoP." For better readability, positive lipoproteins predictions have been highlighted in green. The locus tags BN853_RS03515, BN853_RS05710, BN853_RS06865, BN854_RS05460, ACL_RS00205 that were present in the original table but that do not seem to code for SBPs were removed from this table.

Supplementary Table 8 | List of accessions used in this study for the AYWB_387, AYWB_376, and AYWB_042 families.

Supplementary Table 9 | List of accessions used in this study for RmuC, YneF, AYWB_444, and RNaseY families. One asterisk was added to accessions provided by $\mathrm{nr}$ database of $\mathrm{NCBl}$ and that derive from genes having alternative 
start codons. The translation products obtained with the alternative start codons are listed in Supplementary Material SM16. Doubled-asterisk indicate that the accessions derive from the same gene and differ by the start codon used.

Supplementary Table 10 | List of sequences from the RNaseY family obtained by using an alternative start codon. These accessions are not part of Genbank.

Supplementary Table 11 | List of Amp accessions used in this study.

\section{REFERENCES}

Adams, H., Scotti, P. A., De Cock, H., Luirink, J., and Tommassen, J. (2002). The presence of a helix breaker in the hydrophobic core of signal sequences of secretory proteins prevents recognition by the signal-recognition particle in Escherichia coli. Eur. J. Biochem. 269, 5564-5571. doi: 10.1046/j.1432-1033. 2002.03262.x

Akimaru, J., Matsuyama, S., Tokuda, H., and Mizushima, S. (1991). Reconstitution of a protein translocation system containing purified SecY, SecE, and SecA from Escherichia coli. Proc. Natl. Acad. Sci. U.S.A. 88, 6545-6549. doi: 10.1073/pnas. 88.15.6545

Almagro Armenteros, J. J., Tsirigos, K. D., Sønderby, C. K., Petersen, T. N., Winther, O., Brunak, S., et al. (2019). SignalP 5.0 improves signal peptide predictions using deep neural networks. Nat. Biotechnol. 37, 420-423. doi: 10.1038/s41587-019-0036-z

Ames, G. F., Liu, C. E., Joshi, A. K., and Nikaido, K. (1996). Liganded and unliganded receptors interact with equal affinity with the membrane complex of periplasmic permeases, a subfamily of traffic ATPases. J. Biol. Chem. 271, 14264-14270. doi: 10.1074/jbc.271.24.14264

Anabestani, A., Izadpanah, K., Abbà, S., Galetto, L., Ghorbani, A., Palmano, S., et al. (2017). Identification of putative effector genes and their transcripts in three strains related to "Candidatus Phytoplasma aurantifolia.". Microbiol. Res. 199, 57-66. doi: 10.1016/j.micres.2017.03.001

Arricau-Bouvery, N., Duret, S., Dubrana, M.-P., Batailler, B., Desqué, D., Béven, L., et al. (2018). Variable membrane protein A of flavescence dorée phytoplasma binds the midgut perimicrovillar membrane of euscelidius variegatus and promotes adhesion to its epithelial cells. Appl. Environ. Microbiol. 84:e02487-17. doi: 10.1128/AEM.02487-17

Bagos, P. G., Tsirigos, K. D., Liakopoulos, T. D., and Hamodrakas, S. J. (2008). Prediction of lipoprotein signal peptides in gram-positive bacteria with a hidden markov model. J. Proteome Res. 7, 5082-5093. doi: 10.1021/pr800162c

Bai, X., Correa, V. R., Toruño, T. Y., Ammar, E.-D., Kamoun, S., and Hogenhout, S. A. (2009). AY-WB phytoplasma secretes a protein that targets plant cell nuclei. Mol. Plant Microbe Interact. 22, 18-30. doi: 10.1094/MPMI-221-0018

Bai, X., Zhang, J., Ewing, A., Miller, S. A., Jancso Radek, A., Shevchenko, D. V., et al. (2006). Living with genome instability: the adaptation of phytoplasmas to diverse environments of their insect and plant hosts. J. Bacteriol. 188, 3682-3696. doi: 10.1128/JB.188.10.3682-3696.2006

Baker, J. A., Wong, W.-C., Eisenhaber, B., Warwicker, J., and Eisenhaber, F. (2017). Charged residues next to transmembrane regions revisited: "Positive-inside rule" is complemented by the "negative inside depletion/outside enrichment rule.”. BMC Biol. 15:66. doi: 10.1186/s12915-017-0404-4

Barbara, D. J., Morton, A., Clark, M. F., and Davies, D. L. (2002). Immunodominant membrane proteins from two phytoplasmas in the aster yellows clade (chlorante aster yellows and clover phyllody) are highly divergent in the major hydrophilic region. Microbiology (Reading) 148, 157-167. doi: 10.1099/00221287-148-1-157

Bechhofer, D. H., and Deutscher, M. P. (2019). Bacterial ribonucleases and their roles in RNA metabolism. Crit. Rev. Biochem. Mol. Biol. 54, 242-300. doi: 10.1080/10409238.2019.1651816

Bendtsen, J. D., Nielsen, H., von Heijne, G., and Brunak, S. (2004). Improved prediction of signal peptides: SignalP 3.0. J. Mol. Biol. 340, 783-795. doi: 10. 1016/j.jmb.2004.05.028

Berg, M., Davies, D. L., Clark, M. F., Vetten, H. J., Maie, G., Marcone, C., et al. (1999). Isolation of the gene encoding an immunodominant membrane protein of the apple proliferation phytoplasma, and expression and characterization of the gene product. Microbiology (Reading) 145(Pt 8), 1937-1943. doi: 10.1099/ 13500872-145-8-1937
Supplementary Table 12 | Accessions related to $V m p A, V m p B$, and $V m p 1$, and the associated signal peptide predictions. Positive signal peptide predictions were indicated with "Y," and negative ones with "N" and a gray background for enhanced visibility.

Supplementary Table 13 | List of Imp accessions used in this study.

Supplementary Material 1 | Sequences used in this study, grouped as fasta files.

Boonrod, K., Munteanu, B., Jarausch, B., Jarausch, W., and Krczal, G. (2012). An immunodominant membrane protein (Imp) of 'Candidatus Phytoplasma mali' binds to plant actin. Mol. Plant Microbe Interact. 25, 889-895. doi: 10.1094/ MPMI-11-11-0303

Camacho, C., Coulouris, G., Avagyan, V., Ma, N., Papadopoulos, J., Bealer, K., et al. (2009). BLAST+: architecture and applications. BMC Bioinform. 10:421. doi: 10.1186/1471-2105-10-421

Chang, S. H., Tan, C. M., Wu, C.-T., Lin, T.-H., Jiang, S.-Y., Liu, R.-C., et al. (2018). Alterations of plant architecture and phase transition by the phytoplasma virulence factor SAP11. J. Exp. Bot. 69, 5389-5401. doi: 10.1093/jxb/ery318

Chen, W., Li, Y., Wang, Q., Wang, N., and Wu, Y. (2014). Comparative genome analysis of wheat blue dwarf phytoplasma, an obligate pathogen that causes wheat blue dwarf disease in china. PLoS One 9:e96436. doi: 10.1371/journal. pone.0096436

Cho, S.-T., Kung, H.-J., Huang, W., Hogenhout, S. A., and Kuo, C.-H. (2020). Species boundaries and molecular markers for the classification of $16 \mathrm{SrI}$ phytoplasmas inferred by genome analysis. Front. Microbiol. 11:1531. doi: 10. 3389/fmicb. 2020.01531

Cho, S.-T., Lin, C.-P., and Kuo, C.-H. (2019). Genomic characterization of the periwinkle leaf yellowing (PLY) phytoplasmas in Taiwan. Front. Microbiol. 10:2194. doi: 10.3389/fmicb.2019.02194

Chung, W.-C., Chen, L.-L., Lo, W.-S., Lin, C.-P., and Kuo, C.-H. (2013). Comparative analysis of the peanut witches'-broom phytoplasma genome reveals horizontal transfer of potential mobile units and effectors. PLoS One 8:e62770. doi: 10.1371/journal.pone.0062770

Cimerman, A., Pacifico, D., Salar, P., Marzachì, C., and Foissac, X. (2009). Striking diversity of vmp1, a variable gene encoding a putative membrane protein of the stolbur phytoplasma. Appl. Environ. Microbiol. 75, 2951-2957. doi: 10.1128/ AEM.02613-08

Contreras-Moreira, B., and Vinuesa, P. (2013). GET_HOMOLOGUES, a versatile software package for scalable and robust microbial pangenome analysis. Appl. Environ. Microbiol. 79, 7696-7701. doi: 10.1128/AEM.02411-13

Cranford-Smith, T., and Huber, D. (2018). The way is the goal: how SecA transports proteins across the cytoplasmic membrane in bacteria. FEMS Microbiol. Lett. 365:fny093. doi: 10.1093/femsle/fny093

Edgar, R. C. (2004). MUSCLE: multiple sequence alignment with high accuracy and high throughput. Nucleic Acids Res. 32, 1792-1797. doi: 10.1093/nar/gkh340

Freudl, R. (2018). Signal peptides for recombinant protein secretion in bacterial expression systems. Microb. Cell Fact. 17:52. doi: 10.1186/s12934-018-0901-3

Galetto, L., Bosco, D., Balestrini, R., Genre, A., Fletcher, J., and Marzachì, C. (2011). The major antigenic membrane protein of "Candidatus Phytoplasma asteris" selectively interacts with ATP synthase and actin of leafhopper vectors. PLoS One 6:e22571. doi: 10.1371/journal.pone.0022571

Gouy, M., Guindon, S., and Gascuel, O. (2010). SeaView version 4: a multiplatform graphical user interface for sequence alignment and phylogenetic tree building. Mol. Biol. Evol. 27, 221-224. doi: 10.1093/molbev/msp259

Hamouche, L., Billaudeau, C., Rocca, A., Chastanet, A., Ngo, S., Laalami, S., et al. (2020). Dynamic Membrane localization of RNase Y in Bacillus subtilis. mBio 11:e03337-19. doi: 10.1128/mBio.03337-19

Hogenhout, S. A., Oshima, K., Ammar, E., Kakizawa, S., Kingdom, H. N., and Namba, S. (2008). Phytoplasmas: bacteria that manipulate plants and insects. Mol. Plant Pathol. 9, 403-423. doi: 10.1111/j.1364-3703.2008.00472.x

Hoshi, A., Oshima, K., Kakizawa, S., Ishii, Y., Ozeki, J., Hashimoto, M., et al. (2009). A unique virulence factor for proliferation and dwarfism in plants identified from a phytopathogenic bacterium. Proc. Natl. Acad. Sci. U.S.A 106, 6416-6421. doi: 10.1073/pnas.0813038106

Huang, C.-L., and Ho, K.-C. (2007). Isolation and characterization of the ATPbinding cassette $(\mathrm{ABC})$ transporter system genes from loofah witches' broom phytoplasma. DNA Seq. 18, 347-356. doi: 10.1080/10425170701350784 
Iwabuchi, N., Kitazawa, Y., Maejima, K., Koinuma, H., Miyazaki, A., Matsumoto, O., et al. (2020). Functional variation in phyllogen, a phyllody-inducing phytoplasma effector family, attributable to a single amino acid polymorphism. Mol. Plant Pathol. 21, 1322-1336. doi: 10.1111/mpp.12981

Iwabuchi, N., Maejima, K., Kitazawa, Y., Miyatake, H., Nishikawa, M., Tokuda, R., et al. (2019). Crystal structure of phyllogen, a phyllody-inducing effector protein of phytoplasma. Biochem. Biophys. Res. Commun. 513, 952-957. doi: 10.1016/j.bbrc.2019.04.060

Janik, K., Mithöfer, A., Raffeiner, M., Stellmach, H., Hause, B., and Schlink, K. (2017). An effector of apple proliferation phytoplasma targets TCP transcription factors-a generalized virulence strategy of phytoplasma? Mol. Plant Pathol. 18, 435-442. doi: 10.1111/mpp.12409

Jomantiene, R., Zhao, Y., and Davis, R. E. (2007). Sequence-variable mosaics: composites of recurrent transposition characterizing the genomes of phylogenetically diverse phytoplasmas. DNA Cell Biol. 26, 557-564. doi: $10.1089 /$ dna.2007.0610

Juncker, A. S., Willenbrock, H., von Heijne, G., Brunak, S., Nielsen, H., and Krogh, A. (2003). Prediction of lipoprotein signal peptides in Gram-negative bacteria. Protein Sci. 12, 1652-1662. doi: 10.1110/ps.0303703

Kakizawa, S., Oshima, K., Ishii, Y., Hoshi, A., Maejima, K., Jung, H.-Y., et al. (2009). Cloning of immunodominant membrane protein genes of phytoplasmas and their in planta expression. FEMS Microbiol. Lett. 293, 92-101. doi: 10.1111/j. 1574-6968.2009.01509.x

Kakizawa, S., Oshima, K., Kuboyama, T., Nishigawa, H., Jung, H., Sawayanagi, T., et al. (2001). Cloning and expression analysis of phytoplasma protein translocation genes. Mol. Plant Microbe Interact. 14, 1043-1050. doi: 10.1094/ MPMI.2001.14.9.1043

Kakizawa, S., Oshima, K., and Namba, S. (2006). Diversity and functional importance of phytoplasma membrane proteins. Trends Microbiol. 14, 254-256. doi: 10.1016/j.tim.2006.04.008

Kakizawa, S., Oshima, K., Nishigawa, H., Jung, H.-Y., Wei, W., Suzuki, S., et al. (2004). Secretion of immunodominant membrane protein from onion yellows phytoplasma through the Sec protein-translocation system in Escherichia coli. Microbiology 150, 135-142. doi: 10.1099/mic.0.26521-0

Käll, L., Krogh, A., and Sonnhammer, E. L. L. (2004). A combined transmembrane topology and signal peptide prediction method. J. Mol. Biol. 338, 1027-1036. doi: $10.1016 /$ j.jmb.2004.03.016

Käll, L., Krogh, A., and Sonnhammer, E. L. L. (2007). Advantages of combined transmembrane topology and signal peptide prediction-the Phobius web server. Nucleic Acids Res. 35, W429-W432. doi: 10.1093/nar/gkm256

Kim, A. C., Oliver, D. C., and Paetzel, M. (2008). Crystal structure of a bacterial signal Peptide peptidase. J. Mol. Biol. 376, 352-366. doi: 10.1016/j.jmb.2007.11. 080

Kitazawa, Y., Iwabuchi, N., Himeno, M., Sasano, M., Koinuma, H., Nijo, T., et al. (2017). Phytoplasma-conserved phyllogen proteins induce phyllody across the Plantae by degrading floral MADS domain proteins. J. Exp. Bot. 88, 2799-2811. doi: $10.1093 /$ jxb/erx158

Konnerth, A., Krczal, G., and Boonrod, K. (2016). Immunodominant membrane proteins of phytoplasmas. Microbiology 162, 1267-1273. doi: 10.1099/mic.0. 000331

Krogh, A., Larsson, B., von Heijne, G., and Sonnhammer, E. L. (2001). Predicting transmembrane protein topology with a hidden Markov model: application to complete genomes. J. Mol. Biol. 305, 567-580. doi: 10.1006/jmbi.2000.4315

$\mathrm{Ku}, \mathrm{C}$., Lo, W.-S., and Kuo, C.-H. (2013). Horizontal transfer of potential mobile units in phytoplasmas. Mobile Genetic Elements 3:e26145. doi: 10.4161/mge. 26145

Kube, M., Mitrovic, J., Duduk, B., Rabus, R., and Seemüller, E. (2012). Current view on phytoplasma genomes and encoded metabolism. ScientificWorldJournal 2012:185942. doi: 10.1100/2012/185942

Kube, M., Siewert, C., Migdoll, A. M., Duduk, B., Holz, S., Rabus, R., et al. (2014). Analysis of the complete genomes of Acholeplasma brassicae, A. palmae and A. laidlawii and their comparison to the obligate parasites from "Candidatus Phytoplasma.". J. Mol. Microbiol. Biotechnol. 24, 19-36. doi: 10.1159/00035 4322

Kuhn, A., Koch, H.-G., and Dalbey, R. E. (2017). Targeting and insertion of membrane proteins. EcoSal Plus 7:1128. doi: 10.1128/ecosalplus.ESP-00122016
Larsson, J. (2020). eulerr: Area-Proportional Euler and Venn Diagrams with Ellipses. Available online at: https://cran.r-project.org/package=eulerr (accessed October 16, 2019).

Lee, I. M., Davis, R. E., and Gundersen-Rindal, D. E. (2000). Phytoplasma: phytopathogenic mollicutes. Annu. Rev. Microbiol. 54, 221-255. doi: 10.1146/ annurev.micro.54.1.221

Liao, Y.-T., Lin, S.-S., Lin, S.-J., Sun, W.-T., Shen, B.-N., Cheng, H.-P., et al. (2019). Structural insights into the interaction between phytoplasmal effector causing phyllody 1 (PHYL1) and MADS transcription factor. Plant J. 100, 706-719. doi: $10.1111 /$ tpj. 14463

Lomize, A. L., Lomize, M. A., Krolicki, S. R., and Pogozheva, I. D. (2017). Membranome: a database for proteome-wide analysis of single-pass membrane proteins. Nucleic Acids Res. 45, D250-D255. doi: 10.1093/nar/gkw712

Lu, Y.-T., Cheng, K.-T., Jiang, S.-Y., and Yang, J.-Y. (2014a). Post-translational cleavage and self-interaction of the phytoplasma effector SAP11. Plant Signal Behav. 9:28991. doi: 10.4161/psb.28991

Lu, Y.-T., Li, M.-Y., Cheng, K.-T., Tan, C. M., Su, L.-W., Lin, W.-Y., et al. (2014b). Transgenic plants that express the phytoplasma effector SAP11 Show altered phosphate starvation and defense responses. Plant Physiol. 164, 1456-1469. doi: 10.1104/pp.113.229740

Maclean, A. M., Orlovskis, Z., Kowitwanich, K., Zdziarska, A. M., Angenent, G. C., Immink, R. G. H., et al. (2014). Phytoplasma effector SAP54 hijacks plant reproduction by degrading MADS-box proteins and promotes insect colonization in a RAD23-dependent manner. PLoS Biol. 12:e1001835. doi: 10. 1371/journal.pbio.1001835

MacLean, A. M., Sugio, A., Makarova, O. V., Findlay, K. C., Grieve, V. M., Tóth, R., et al. (2011). Phytoplasma effector SAP54 induces indeterminate leaf-like flower development in Arabidopsis plants. Plant Physiol. 157, 831-841. doi: 10.1104/pp.111.181586

Maejima, K., Iwai, R., Himeno, M., Komatsu, K., Kitazawa, Y., Fujita, N., et al. (2014). Recognition of floral homeotic MADS domain transcription factors by a phytoplasmal effector, phyllogen, induces phyllody. Plant J. 78, 541-554. doi: 10.1111/tpj.12495

Maejima, K., Kitazawa, Y., Tomomitsu, T., Yusa, A., Neriya, Y., Himeno, M., et al. (2015). Degradation of class E MADS-domain transcription factors in Arabidopsis by a phytoplasmal effector, phyllogen. Plant Signal Behav. 10:e1042635. doi: 10.1080/15592324.2015.1042635

Malembic-Maher, S., Desqué, D., Khalil, D., Salar, P., Bergey, B., Danet, J.-L., et al. (2020). When a Palearctic bacterium meets a Nearctic insect vector: Genetic and ecological insights into the emergence of the grapevine Flavescence dorée epidemics in Europe. PLoS Pathog. 16:e1007967. doi: 10.1371/journal.ppat. 1007967

Minato, N., Himeno, M., Hoshi, A., Maejima, K., Komatsu, K., Takebayashi, Y., et al. (2014). The phytoplasmal virulence factor TENGU causes plant sterility by downregulating of the jasmonic acid and auxin pathways. Sci. Rep. 4:7399. doi: 10.1038/srep07399

Music, M. S., Samarzija, I., Hogenhout, S. A., Haryono, M., Cho, S.-T., and Kuo, C.-H. (2018). The genome of "Candidatus Phytoplasma solani" strain SA-1 is highly dynamic and prone to adopting foreign sequences. Syst. Appl. Microbiol. 42, 117-127. doi: 10.1016/j.syapm.2018.10.008

Narita, S.-I., and Tokuda, H. (2017). Bacterial lipoproteins; biogenesis, sorting and quality control. Biochim. Biophys. Acta Mol. Cell Biol. Lipids 1862, 1414-1423. doi: 10.1016/j.bbalip.2016.11.009

Natale, P., Brüser, T., and Driessen, A. J. M. (2008). Sec- and Tat-mediated protein secretion across the bacterial cytoplasmic membrane-distinct translocases and mechanisms. Biochim. Biophys. Acta 1778, 1735-1756. doi: 10.1016/j.bbamem. 2007.07.015

NCBI Resource Coordinators (2018). Database resources of the national center for biotechnology information. Nucleic Acids Res. 46, D8-D13. doi: 10.1093/nar/ gkx1095

Neriya, Y., Sugawara, K., Maejima, K., Hashimoto, M., Komatsu, K., Minato, N., et al. (2011). Cloning, expression analysis, and sequence diversity of genes encoding two different immunodominant membrane proteins in poinsettia branch-inducing phytoplasma (PoiBI). FEMS Microbiol. Lett. 324, 38-47. doi: 10.1111/j.1574-6968.2011.02384.x

Nielsen, H. (2017). Predicting secretory proteins with SignalP. Methods Mol. Biol. 1611, 59-73. doi: 10.1007/978-1-4939-7015-5_6 
Nielsen, H., Engelbrecht, J., Brunak, S., and von Heijne, G. (1997). Identification of prokaryotic and eukaryotic signal peptides and prediction of their cleavage sites. Protein Eng. 10, 1-6. doi: 10.1093/protein/10.1.1

Nielsen, H., and Krogh, A. (1998). Prediction of signal peptides and signal anchors by a hidden markov model. Proc. Int. Conf. Intell. Syst. Mol. Biol. 6, $122-130$.

Nielsen, H., Tsirigos, K. D., Brunak, S., and von Heijne, G. (2019). A brief history of protein sorting prediction. Protein J. 38, 200-216. doi: 10.1007/s10930-01909838-3

Orlovskis, Z., and Hogenhout, S. A. (2016). A bacterial parasite effector mediates insect vector attraction in host plants independently of developmental changes. Front. Plant Sci. 7:885. doi: 10.3389/fpls.2016.00885

Paetzel, M. (2014). Structure and mechanism of Escherichia coli type I signal peptidase. Biochim. Biophys. Acta (BBA) Mol. Cell Res. 1843, 1497-1508. doi: 10.1016/j.bbamcr.2013.12.003

Pecher, P., Moro, G., Canale, M. C., Capdevielle, S., Singh, A., MacLean, A., et al. (2019). Phytoplasma SAP11 effector destabilization of TCP transcription factors differentially impact development and defence of Arabidopsis versus maize. PLoS Pathog. 15:e1008035. doi: 10.1371/journal.ppat.1008035

Petersen, T. N., Brunak, S., von Heijne, G., and Nielsen, H. (2011). SignalP 4.0: discriminating signal peptides from transmembrane regions. Nat. Methods 8 , 785-786. doi: 10.1038/nmeth.1701

Quaglino, F., Kube, M., Jawhari, M., Abou-Jawdah, Y., Siewert, C., Choueiri, E., et al. (2015). "Candidatus Phytoplasma phoenicium" associated with almond witches'-broom disease: from draft genome to genetic diversity among strain populations. BMC Microbiol. 15:148. doi: 10.1186/s12866-015-0487-4

Renaudin, J., Béven, L., Batailler, B., Duret, S., Desqué, D., Arricau-Bouvery, N., et al. (2015). Heterologous expression and processing of the flavescence dorée phytoplasma variable membrane protein VmpA in Spiroplasma citri. BMC Microbiol. 15:82. doi: 10.1186/s12866-015-0417-5

Saccardo, F., Martini, M., Palmano, S., Ermacora, P., Scortichini, M., Loi, N., et al. (2012). Genome drafts of four phytoplasma strains of the ribosomal group 16SrIII. Microbiology 158, 2805-2814. doi: 10.1099/mic.0.061432-0

Saito, A., Hizukuri, Y., Matsuo, E., Chiba, S., Mori, H., Nishimura, O., et al. (2011). Post-liberation cleavage of signal peptides is catalyzed by the site-2 protease (S2P) in bacteria. Proc. Natl. Acad. Sci. U.S.A. 108, 13740-13745. doi: 10.1073/pnas.1108376108

Scheepers, G. H., Lycklama, A., Nijeholt, J. A., and Poolman, B. (2016). An updated structural classification of substrate-binding proteins. FEBS Lett. 590, 4393-4401. doi: 10.1002/1873-3468.12445

Serebryakova, M. V., Demina, I. A., Galyamina, M. A., Kondratov, I. G., Ladygina, V. G., and Govorun, V. M. (2011). The acylation state of surface lipoproteins of mollicute Acholeplasma laidlawii. J. Biol. Chem. 286, 22769-22776. doi: 10. 1074/jbc.M111.231316

Siampour, M., Izadpanah, K., Galetto, L., Salehi, M., and Marzachi, C. (2013). Molecular characterization, phylogenetic comparison and serological relationship of the Imp protein of several "Candidatus Phytoplasma aurantifolia” strains. Plant Pathol. 62, 452-459. doi: 10.1111/j.1365-3059.2012. 02662.x

Sievers, F., Wilm, A., Dineen, D., Gibson, T. J., Karplus, K., Li, W., et al. (2011). Fast, scalable generation of high-quality protein multiple sequence alignments using Clustal Omega. Mol. Syst. Biol. 7:539. doi: 10.1038/msb.2011.75

Siewert, C., Hess, W. R., Duduk, B., Huettel, B., Reinhardt, R., Büttner, C., et al. (2014a). Complete genome determination and analysis of Acholeplasma oculi strain 19L, highlighting the loss of basic genetic features in the Acholeplasmataceae. BMC Genom. 15:931. doi: 10.1186/1471-2164-15-931

Siewert, C., Luge, T., Duduk, B., Seemuller, E., Buttner, C., Sauer, S., et al. (2014b). Analysis of expressed genes of the bacterium "Candidatus Phytoplasma Mali" highlights key features of virulence and metabolism. PLoS One 9:e94391. doi: 10.1371/journal.pone.0094391

Slupska, M. M., Chiang, J. H., Luther, W. M., Stewart, J. L., Amii, L., Conrad, A., et al. (2000). Genes involved in the determination of the rate of inversions at short inverted repeats. Genes Cells 5, 425-437. doi: 10.1046/j.1365-2443.2000. 00341.x
Sparks, M. E., Bottner-Parker, K. D., Gundersen-Rindal, D. E., and Lee, I.-M. (2018). Draft genome sequence of the New Jersey aster yellows strain of 'Candidatus Phytoplasma asteris.'. PLoS One 13:e0192379. doi: 10.1371/journal. pone.0192379

Strohmayer, A., Moser, M., Si-Ammour, A., Krczal, G., and Boonrod, K. (2019). 'Candidatus Phytoplasma mali' genome encodes a protein that functions as an E3 ubiquitin ligase and could inhibit plant basal defense. MPMI 32, 1487-1495. doi: 10.1094/MPMI-04-19-0107-R

Sugawara, K., Honma, Y., Komatsu, K., Himeno, M., Oshima, K., and Namba, S. (2013). The alteration of plant morphology by small peptides released from the proteolytic processing of the bacterial peptide TENGU. Plant Physiol. 162, 2005-2014. doi: 10.1104/pp.113.218586

Sugio, A., and Hogenhout, S. A. (2012). The genome biology of phytoplasma: modulators of plants and insects. Curr. Opin. Microbiol. 15, 247-254. doi: 10.1016/j.mib.2012.04.002

Sugio, A., Kingdom, H. N., Maclean, A. M., Grieve, V. M., and Hogenhout, S. A. (2011). Phytoplasma protein effector SAP11 enhances insect vector reproduction by manipulating plant development and defense hormone biosynthesis. Proc. Natl. Acad. Sci. U.S.A 108, E1254-E1263. doi: 10.1073/pnas. 1105664108

Suzuki, S., Oshima, K., Kakizawa, S., Arashida, R., Jung, H.-Y., Yamaji, Y., et al. (2006). Interaction between the membrane protein of a pathogen and insect microfilament complex determines insect-vector specificity. Proc. Natl. Acad. Sci. U.S.A. 103, 4252-4257. doi: 10.1073/pnas. 0508668103

Tan, C. M., Li, C.-H., Tsao, N.-W., Su, L.-W., Lu, Y.-T., Chang, S. H., et al. (2016). Phytoplasma SAP11 alters 3-isobutyl-2-methoxypyrazine biosynthesis in Nicotiana benthamiana by suppressing NbOMT1. J. Exp. Bot. 67, 4415-4425. doi: $10.1093 /$ jxb/erw225

Tsirigotaki, A., De Geyter, J., Šoštaric, N., Economou, A., and Karamanou, S. (2017). Protein export through the bacterial Sec pathway. Nat. Rev. Microbiol. 15, 21-36. doi: 10.1038/nrmicro.2016.161

van der Heide, T., and Poolman, B. (2002). ABC transporters: one, two or four extracytoplasmic substrate-binding sites? EMBO Rep. 3, 938-943. doi: 10.1093/ embo-reports/kvf201

Wang, J., Song, L., Jiao, Q., Yang, S., Gao, R., Lu, X., et al. (2018a). Comparative genome analysis of jujube witches'-broom Phytoplasma, an obligate pathogen that causes jujube witches'-broom disease. BMC Genom. 19:689. doi: 10.1186/ s12864-018-5075-1

Wang, N., Li, Y., Chen, W., Yang, H. Z., Zhang, P. H., and Wu, Y. F. (2018b). Identification of wheat blue dwarf phytoplasma effectors targeting plant proliferation and defence responses. Plant Pathol. 67, 603-609. doi: 10.1111/ ppa. 12786

Wang, N., Yang, H., Yin, Z., Liu, W., Sun, L., and Wu, Y. (2018c). Phytoplasma effector SWP1 induces witches' broom symptom by destabilizing the TCP transcription factor BRANCHED1. Mol. Plant Pathol. 19:2623. doi: 10.1111/ mpp. 12733

Waterhouse, A. M., Procter, J. B., Martin, D. M. A., Clamp, M., and Barton, G. J. (2009). Jalview Version 2-a multiple sequence alignment editor and analysis workbench. Bioinformatics 25, 1189-1191. doi: 10.1093/bioinformatics/btp033

Wei, W., Kakizawa, S., Jung, H.-Y., Suzuki, S., Tanaka, M., Nishigawa, H., et al. (2004). An antibody against the SecA membrane protein of one phytoplasma reacts with those of phylogenetically different phytoplasmas. Phytopathology 94 , 683-686. doi: 10.1094/PHYTO.2004.94.7.683

Conflict of Interest: The authors declare that the research was conducted in the absence of any commercial or financial relationships that could be construed as a potential conflict of interest.

Copyright (c) 2021 Garcion, Béven and Foissac. This is an open-access article distributed under the terms of the Creative Commons Attribution License (CC BY). The use, distribution or reproduction in other forums is permitted, provided the original author(s) and the copyright owner(s) are credited and that the original publication in this journal is cited, in accordance with accepted academic practice. No use, distribution or reproduction is permitted which does not comply with these terms. 